\title{
Misoprostol regulates Bnip3 repression and alternative splicing to control cellular calcium homeostasis during hypoxic stress
}

\author{
Jared T. Field ${ }^{1,2}$, Matthew D. Martens ${ }^{1,2}$, Wajihah Mughal ${ }^{1,2}$, Yan Hai ${ }^{2}$, Donald Chapman², Grant M. Hatch ${ }^{2,3,4}$, \\ Tammy L. Ivanco ${ }^{2,5}$, William Diehl-Jones ${ }^{2,6,7}$ and Joseph W. Gordon (1) $1,2,8$
}

\begin{abstract}
The cellular response to hypoxia involves the activation of a conserved pathway for gene expression regulated by the transcription factor complex called hypoxia-inducible factor (HIF). This pathway has been implicated in both the adaptive response to hypoxia and in several hypoxic-ischemic-related pathologies. Perinatal hypoxic injury, often associated with prematurity, leads to multi-organ dysfunction resulting in significant morbidity and mortality. Using a rodent model of neonatal hypoxia and several representative cell lines, we observed HIF1a activation and downstream induction of the cell death gene Bnip3 in brain, large intestine, and heart which was mitigated by administration of the prostaglandin E1 analog misoprostol. Mechanistically, we determined that misoprostol inhibits full-length Bnip3 (Bnip3-FL) expression through PKA-mediated NF-KB (P65) nuclear retention, and the induction of prosurvival splice variants. We observed that the dominant small pro-survival variant of Bnip3 in mouse cells lacks the third

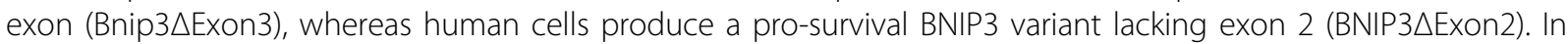
addition, these small Bnip3 splice variants prevent mitochondrial dysfunction, permeability transition, and necrosis triggered by Bnip3-FL by blocking calcium transfer from the sarco/endoplasmic reticulum to the mitochondria. Furthermore, misoprostol and Bnip3 $\triangle$ Exon3 promote nuclear calcium accumulation, resulting in HDAC5 nuclear export, NFAT activation, and adaptive changes in cell morphology and gene expression. Collectively, our data suggests that misoprostol can mitigate the potential damaging effects of hypoxia on multiple cell types by activating adaptive cell survival pathways through Bnip3 repression and alternative splicing.
\end{abstract}

\section{Introduction}

Hypoxia is a central element in many diseases of prematurity, including hypoxic/ischemic encephalopathy $(\mathrm{HIE})^{1}$, necrotizing enterocolitis (NEC) $)^{2}$, retinopathy of prematurity $^{3}$, and persistent pulmonary hypertension of the newborn $(\mathrm{PPHN})^{4}$. In addition, cardiac dysfunction is an important predictor of morbidity and mortality in hypoxia- and asphyxia-related neonatal disorders, as

\footnotetext{
Correspondence: Joseph W. Gordon (joseph.gordon@umanitoba.ca) ${ }^{1}$ Department of Human Anatomy and Cell Science, University of Manitoba, Winnipeg, Canada

${ }^{2}$ Children's Hospital Research Institute of Manitoba, Winnipeg, Canada Full list of author information is available at the end of the article. These authors contributed equally: Jared T. Field, Matthew D. Martens Edited by A. Rufini
}

impaired cardiac metabolism and contractile performance compromise tissue perfusion ${ }^{5,6}$. Regardless of the cause, oxygen-deprived cells display accumulating levels of transcription factors belonging to the hypoxia-inducible factor-alpha (HIF $\alpha$ ) family. During normoxia, HIF $\alpha$ is hydroxylated within its oxygen degradation domain (ODD) by the prolyl-hydroxylase domain (PHD) enzymes, triggering HIF $\alpha$ degradation by the proteasome ${ }^{7}$. However, a reduced cellular oxygen tension inhibits the activity of the PHD enzymes, allowing HIF $\alpha$ to accumulate in the nucleus and activate transcription through dimerization with the HIF $\beta$ (i.e., ARNT) subunit ${ }^{7}$. Although cell-type specific differences in this pathway

\section{(c) 2018 The Author(s)}

(c) Open Access This article is licensed under a Creative Commons Attribution 4.0 International License, which permits use, sharing, adaptation, distribution and reproduction in any medium or format, as long as you give appropriate credit to the original author(s) and the source, provide a link to the Creative Commons license, and indicate if changes were made. The images or other third party material in this article are included in the article's Creative Commons license, unless indicated otherwise in a credit line to the material. If material is not included in the article's Creative Commons license and your intended use is not permitted by statutory regulation or exceeds the permitted use, you will need to obtain permission directly from the copyright holder. To view a copy of this license, visit http://creativecommons.org/licenses/by/4.0/. 
exist, there is a remarkable conservation amongst multiple cell-types in response to HIF $\alpha$ activation, including the resulting induction in glycolytic metabolism and the reduction of mitochondrial respiration ${ }^{7,8}$.

HIF1 $\alpha$ has been shown to increase the expression of members of the Bcl-2 gene family, including the BCL-2/ adenovirus E1B $19 \mathrm{kD}$-interacting protein 3 (Bnip3), whose protein product plays a pivotal role in hypoxiainduced apoptosis, necrosis, and autophagy ${ }^{9,10}$. Depending on the cellular context, Bnip3 has been previously shown to induce macro-autophagy by disrupting the Beclin-1/Bcl-2 complex ${ }^{11}$, promote mitochondrial outer membrane permeability (MOMP) leading to apopto$\operatorname{sis}^{12,13}$, and trigger mitochondrial permeability transitiondependent necrosis by releasing calcium from the endoplasmic reticulum ${ }^{12,14}$. In cardiomyocytes, Bnip3 expression is negatively regulated by a p65/p50 dimer of the NF$\kappa \mathrm{B}$ family (reviewed by Gordon et al. ${ }^{15}$ ). Although canonical NF- $\kappa B$ signaling occurs through repression of Inhibitor of $\kappa \mathrm{B}(\mathrm{I} \kappa \mathrm{B})$ by the I $\mathrm{KB}$ kinase (IKK), other signaling pathways have been shown to alter NF- $\mathrm{kB}$ transcriptional activity, co-factor interaction, and alter the nuclear-tocytoplasmic shuttling of the p65 subunit ${ }^{16,17}$. For example, PKA phosphorylates human P65 at Serine-276 to promote nuclear accumulation and the interaction with the histone acetyl transferase $\mathrm{p} 300^{18-20}$. However, in the context of the Bnip3 promoter, p65 serves to recruit HDAC1 to repress gene expression ${ }^{15}$.

Bnip3 has been shown to be alternatively spliced leading to the production of an endogenous inhibitor that lacks the third exon, called Bnip $3 \Delta$ Exon $3^{21}$. The fusion of exon 2 to exon 4 of the Bnip3 gene results in a frame-shift, a premature stop codon, and the production of a truncated protein with a divergent C-terminus. Bnip3 $\Delta$ Exon3 appears to act as an endogenous inhibitor of full-length Bnip3 (Bnip3-FL) by preventing mitochondrial depolarization, and promoting cell viability ${ }^{21}$. However, the precise mechanism(s) by which Bnip3$\Delta$ Exon3 inhibits hypoxia- and Bnip3-induced cell death remain less clear.

Recently, we demonstrated that Bnip3 expression was elevated in enterocytes subjected to nutrient/oxidative stress induced by breast milk fortifiers, while Bnip3induced enterocyte cell death was inhibited by exogenous expression of Bnip $3 \Delta \mathrm{Exon}^{22}$. Furthermore, fortifierinduced cellular toxicity was completely abrogated by treatment of enterocytes with the prostaglandin analog misoprostol ${ }^{22}$. These compelling findings led us to investigate whether misoprostol could protect cells against hypoxia-induced injury. Furthermore, given the degree of conservation in the cellular response to hypoxia, we sought to determine if misoprostol could protect multiple cell types from Bnip3-induced injury, such as that occurring during neonatal hypoxia/asphyxia.
In this report, we provide evidence that misoprostol opposes hypoxia-induced Bnip3 expression in multiple tissues, including gut, brain, and the heart. In cultured cells, we observed that misoprostol activates PKA signaling and promotes nuclear localization of P65 to suppress Bnip3-FL expression and increase the expression of smaller splice variants. In addition, we discovered a previously unidentified Bnip3 splice variant lacking exon 2 (BNIP3 $\Delta$ Exon2), which is expressed in human cells. Remarkably, this splice variant contains the same frameshift as mouse Bnip3 $\Delta$ Exon3, resulting in a conserved Cterminal amino acid sequence. Mechanistically, we determined that a combination of NF- $\mathrm{KB}$ and HIF1 $\alpha$ activation alters BNIP3 splicing and induces the expression of the smaller variants. Finally, we experimentally altered the ratio of Bnip3-FL to Bnip3 $\Delta$ Exon3, and Bnip3FL to BNIP3 $\triangle$ Exon2 in multiple cell types, and observed that the smaller variants prevent mitochondrial permeability transition and cell death by inhibiting mitochondrial calcium accumulation and promoting nuclear calcium-dependent cell adaptation. These findings suggest that prostaglandin signaling may play an important developmental role that can be used therapeutically to circumvent many of the morbidities associated with neonatal hypoxia.

\section{Results \\ Misoprostol inhibits Bnip3-FL expression in vivo}

Previously, we demonstrated that misoprostol could inhibit cell death in cultured enterocytes exposed to nutrient stress ${ }^{22}$. Moreover, our previous work implicated the hypoxia-inducible gene Bnip3 in this process. In order to further these studies in vivo, we exposed neonatal rats to 7 days of hypoxia ( $10 \%$ oxygen), prior to weaning, with and without misoprostol treatment $(10 \mu \mathrm{g} / \mathrm{kg}$; subcutaneous). This hypoxia protocol has been previously shown to induce cognitive impairment consistent with $\mathrm{HIE}^{23,24}$. Analysis of Bnip3 expression by western blot demonstrated increased Bnip3-FL expression in the hippocampus, large intestine, and heart of hypoxia-exposed animals, which was completely abrogated by concurrent misoprostol treatment (Fig. 1a).

To mechanistically dissect the cellular signaling pathway regulating Bnip3 repression following misoprostol treatment, we used HCT-116 cells as this cell line has low background expression of cell-type specific marker genes, but expresses prostaglandin receptors such as $\mathrm{EP} 4^{25}$. In addition, canonical NF- $\mathrm{kB}$ signaling has been previously shown to be a critical regulator of Bnip3 expression during hypoxia (reviewed by Gordon et al. ${ }^{15}$ ). Moreover, protein kinase-A (PKA) is a known down-stream effector of the prostaglandin receptor EP4 and has been shown to phosphorylate P65 of the NF-kB complex at Serine-276 to promote nuclear accumulation ${ }^{18}$. Thus, we tested the 


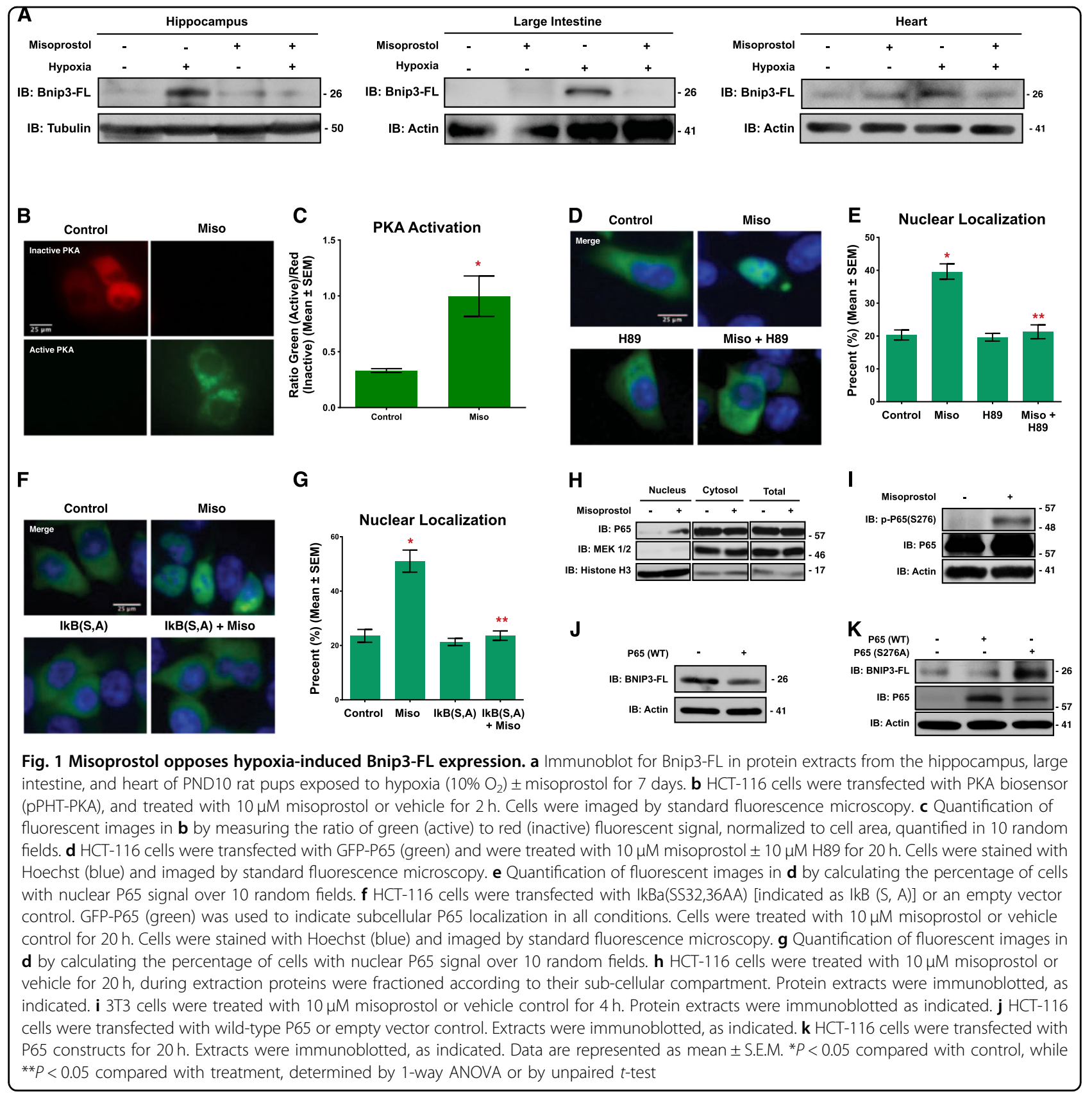

hypothesis that misoprostol could repress Bnip3-FL expression through a conserved PKA- and NF- $\mathrm{B}$ dependent mechanism. Using a genetically-encoded plasmid-based PKA biosensor (pPHT-PKA) ${ }^{26}$, we observed marked PKA activation in cells treated with misoprostol (Fig. 1b, c). Consistent with our hypothesis, we observed increased nuclear localization of a human P65-GFP construct in misoprostol treated cells (Fig. 1d, e). However, when cells were co-treated with misoprostol and the PKA-inhibitor H89, this effect was lost and P65 was retained in the cytoplasm. To further investigate the role of NF- $\mathrm{kB}$ signaling in this misoprostol-induced response, we expressed the same P65-GFP construct concurrently with a dominant negative form of the Inhibitor of kappa-B (IкB S32A, S36A, or IkB-SA). Here, we observed that the misoprostol-induced nuclear localization was lost in the presence of IкB-SA (Fig. 1f, g). Additionally, we performed nuclear and cytosolic fractionation studies to biochemically confirm the observed phenomenon of P65-nuclear localization. We observed that misoprostol treatment had no effect on total P65 expression (Fig. 1h). However, consistent with our P65-GFP findings, we observed an increase in levels of nuclear P65. Next, we assessed the role of misoprostol in 


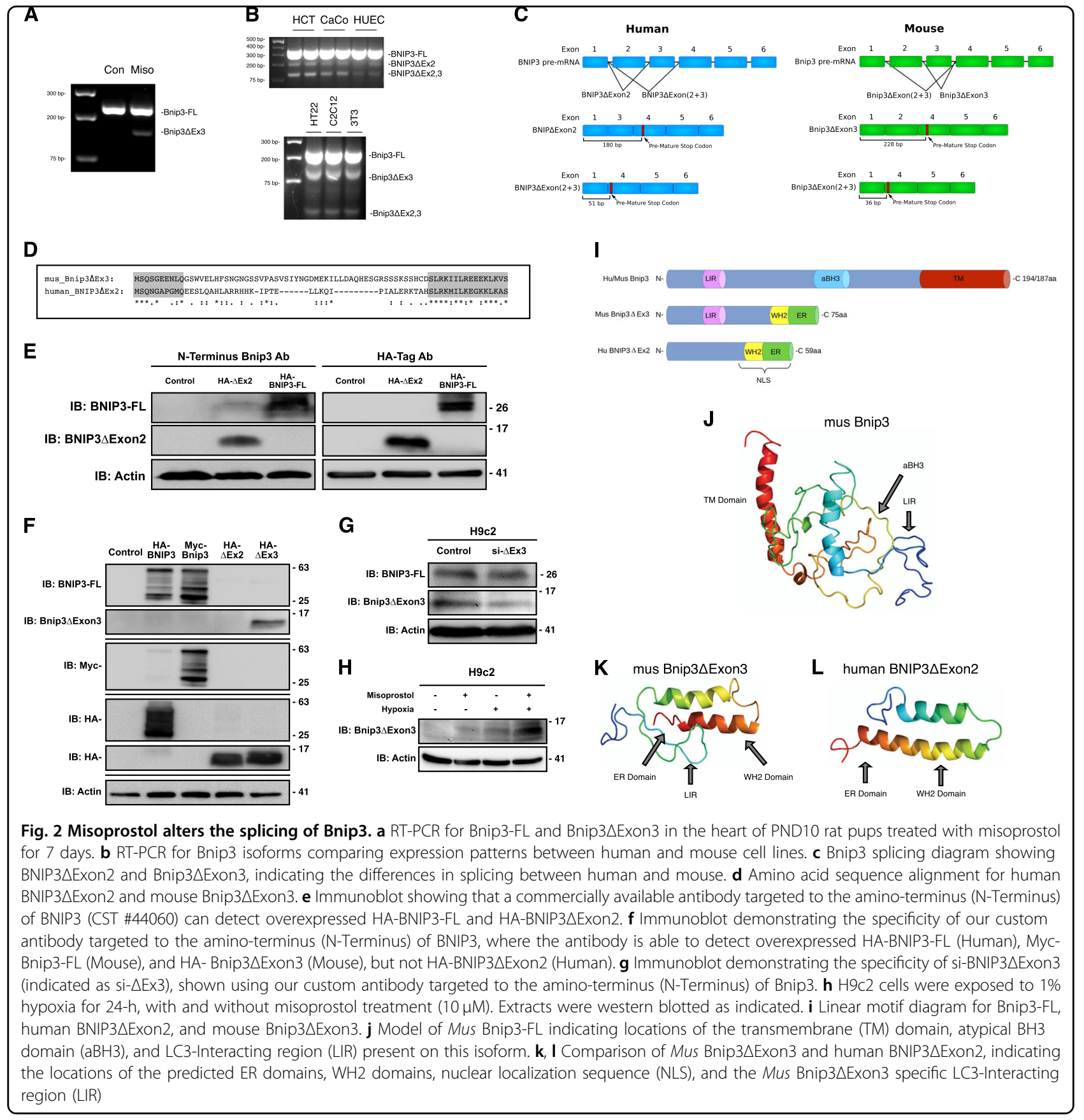

the regulation of $\mathrm{P} 65$ phosphorylation at a known PKA phosphorylation site, serine-276. Shown in Fig. 1i, we observed that misoprostol treatment increased phosphop65. The migration of this band is consistent with information provided by the manufacturer.

To confirm that NF- $\mathrm{kB}$ regulates BNIP3-FL expression, we expressed P65 and analyzed BNIP3-FL expression by western blot. Ectopic expression of P65 reduced BNIP3FL protein levels (Fig. 1j). Furthermore, we expressed wild-type P65, and a P65 mutant form, where the PKA phospho-acceptor site, Ser-276, was mutated to a neutral alanine (P65-S276A) thereby ablating phosphorylation at this site. This experiment confirmed that wild-type P65 represses BNIP3-FL expression; however, when Ser-276 is neutralized, BNIP3-FL expression is enhanced (Fig. 1k).

Collectively, these findings support a model of BNIP3FL repression where misoprostol activates PKA signaling to promote P65 phosphorylation at the Ser-276 site, leading to nuclear accumulation of the NF- $\mathrm{KB}$ complex and subsequent repression of BNIP3-FL expression. 


\section{Discovery of BNIP3 $\Delta$ Exon2}

Recently, it was reported that the rat Bnip3 pre-mRNA undergoes alternative splicing to yield a full-length protein containing all six exons, and a small splice variant lacking the third exon (Bnip3 $\Delta$ Exon3) ${ }^{21}$. Moreover, the fusion of exon 2 to exon 4 in the Bnip3 $\triangle$ Exon3 mRNA results in a frame-shift and the generation of a pre-mature stop codon. Functionally, Bnip3 $\Delta$ Exon3 acts as an endogenous inhibitor of Bnip3-FL, blocking hypoxia- and nutrient stress-induced cell death ${ }^{21,22}$. Based on our findings that misoprostol suppressed Bnip3-FL expression elicited by neonatal hypoxia, we used semi-quantitative RT-PCR to evaluate whether misoprostol could alter Bnip3 splicing. Using primers that spanned the exon 2-4 junction, we detected a smaller amplicon representing Bnip3 $\Delta$ Exon3 in RNA isolated from hearts of neonatal rats treated with misoprostol, but not in vehicle-treated animals (Fig. 2a).

To our knowledge, Bnip3 $\Delta$ Exon3 expression has not been reported at the protein level. Recently, a commercially available antibody targeting exon 1 of BNIP3 has come to market. This antibody could hypothetically detect smaller BNIP3 variants; however, this antibody only detects human BNIP3 protein (Cell Signaling Technology; \#44060). In order to evaluate the role of misoprostol promoting BNIP3 splicing in human cell lines, we attempted to clone human BNIP3 $\triangle$ Exon3. We designed a forward primer encompassing the $5^{\prime}$-ATG of human $B N I P 3$ and a reverse primer containing the premature stop codon identified in mouse Bnip $3 \Delta$ Exon3. Restriction enzyme sites were incorporated into these primers to allow easy ligation into pcDNA3 and sequencing. Interestingly, in three distinct human cells lines we observed three amplicons generated by these primers (Fig. 2b). Based on sequencing analysis, the largest amplicon represents a fragment of BNIP3-FL. However, the middle and smallest amplicons represent splice variants lacking exon 2 (BNIP3 $\triangle$ Exon2) and exons 2 and 3 (BNIP3 $\Delta$ Exon2 $+3)$, respectively. Using the same approach in three representative mouse cell lines, we identified a fragment of Bnip3-FL, Bnip3 $\Delta$ Exon3, and Bnip3 $\Delta$ Exon2 +3 (Fig. 2b). These findings suggest that Bnip3 is differentially spliced in human versus rodent cells (Fig. 2c). The variants with two missing exons form very small peptides due to early stop codons in their reading frame (Fig. 2c and Supplemental). Interestingly, the mouse Bnip3$\Delta$ Exon3 and human BNIP3 $\Delta$ Exon2 share the same frameshift, and generate a conserved $\mathrm{C}$-terminus when translated (Fig. 2d) (Alignment performed on the CLUSTAL O 1.2.4 multiple sequence alignment tool). Importantly, when expressed in HCT-116 cells, we were able to detect both human HA-BNIP3-FL and HA-BNIP3$\triangle$ Exon2 with the commercially available $\mathrm{N}$-terminal BNIP3 antibody (Fig. 2e), suggesting that detection of endogenous protein of these small BNIP3 splice variants is possible in human cells. However, this commerciallyavailable antibody could not detect mouse Bnip $3 \Delta$ Exon3 protein. To solve this, we designed a custom Bnip3 antibody specifically targeted to a region of the $\mathrm{N}$-terminus of full-length Bnip3 that is conserved in humans and rodents. We then performed a series of validation experiments to test this custom antibody. First, we tested if the antibody was able to detect HA-BNIP3-FL, mycBnip3-FL, HA-BNIP3 $\triangle$ Exon2, or HA-Bnip3 $\triangle$ Exon3 in a side-by-side comparison with commercial HA-tag or myc-tag antibodies. As shown in Fig. 2f, the antibody successfully detects both human and mouse full-length BNIP3/Bnip3, and what is likely the monomeric and dimeric forms at the approximate sizes of 30 and $60 \mathrm{kDa}$, respectively. Importantly, we could detect the smaller mouse isoform, Bnip3 $\Delta$ Exon3, but not the human isoform BNIP3 $\triangle$ Exon2, as this epitope spans the second exon in the human gene. Second, we used an siRNA targeted to the unique sequence of the Exon 2-4 junction of Bnip3 $\Delta$ Exon3 to knockdown endogenous Bnip3 $\Delta$ Exon3 protein in H9c2 cells (Fig. 2g). This set of experiments provides evidence that both the custom antibody successfully detects Bnip $3 \Delta$ Exon3, and that the siRNA knockdown shows specificity for Bnip $3 \Delta$ Exon 3 and has little effect on the expression of full-length Bnip3 protein. Finally, we exposed H9c2 cells to 24-h of hypoxia, with and without misoprostol treatment and performed western blots for endogenous Bnip3 $\Delta$ Exon3 (Fig. 2h). We observed a modest induction of Bnip3 $\Delta$ Exon3 with both misoprostol and hypoxia, and a more robust increase in Bnip3 $\Delta$ Exon3 expression with combined treatment. To our knowledge this is the first documentation of Bnip3$\Delta$ Exon3 detected as a protein; furthermore, we demonstrate that endogenous expression of this protein is enhanced by misoprostol treatment in a rodent cardiac cell line.

In silico domain mapping of the smaller Bnip3 variants revealed that neither mouse Bnip3 $\Delta$ Exon3 nor human BNIP3 $\triangle$ Exon2 contain the atypical BH3 domain of the full-length splice variants, and only mouse Bnip3 $\Delta$ Exon3 retained the $\mathrm{N}$-terminal LC3 interacting region (LIR) (Fig. 2h). Interestingly, both mouse Bnip3 $\Delta$ Exon3 and human BNIP3 $\triangle$ Exon2 contain C-terminal WH2 actinbinding motifs and a di-lysine ER retention signal (ER). Moreover, the C-terminal region of human BNIP3 $\triangle$ Exon2 contains a predicted bipartite nuclear localizing signal (NLS). Finally, the Phyre2 (Protein Homology/analogY Recognition Engine V 2.0) web portal for protein modeling provided the predicted structure of the smaller Bnip3 splice variants, described previously ${ }^{27,28}$. We used the predicted structure of mouse Bnip3-FL for comparison (Fig. 2i). Intriguingly, the Phyre2 portal predicted a remarkably similar structure for both mouse 
Bnip3 $\Delta$ Exon3 and human BNIP3 $\Delta$ Exon2 (Fig. 2j, k), which supports the idea that they may function in a similar biological manner.

\section{HIF1a and NF-KB P65 regulate BNIP3 expression and splicing}

To further investigate how the expression of the smaller BNIP3 variants are regulated, we treated HCT-116 with misoprostol, and the HIF $\alpha$ stabilizer cobalt chloride $\left(\mathrm{CoCl}_{2}\right)$ for 16-h. Using semi-quantitative RT-PCR and primers designed to detect both BNIP3-FL and BNIP3$\Delta$ Exon2, we observed that misoprostol alone reduced BNIP3-FL expression, while $\mathrm{CoCl}_{2}$ treatment induced the expression of both BNIP3 splice variants (Fig. 3a). However, in the presence of misoprostol, we observed proportionally more BNIP3 $\triangle$ Exon2. Next, we performed the same experiment using the N-terminal BNIP3 antibody, combined with western blot analysis, and observed induction of BNIP3-FL concurrent with increased HIF1 $\alpha$ when cells were treated with $\mathrm{CoCl}_{2}$ (Fig. 3b). When combined with misoprostol, $\mathrm{CoCl}_{2}$ induced the expression of a $11-17 \mathrm{kDa}$ band as detected by the $\mathrm{N}$-terminal BNIP3 antibody, consistent with migration pattern of BNIP3 $\triangle$ Exon2 observed in Fig. 2e. Interestingly, at this dose and time point misoprostol did not affect BNIP3-FL protein expression. To reconcile this observation with our misoprostol findings in vivo, we extended our time-course and treated cells with $\mathrm{CoCl}_{2}$ and misoprostol for 36-h. At this time point, misoprostol attenuated $\mathrm{CoCl}_{2}$-induced BNIP3-FL expression, while the expression BNIP3 $\Delta$ Exon2 was less effected (Fig. 3c). These findings suggest that misoprostol alters BNIP3 splicing at earlier time points, then represses BNIP3-FL expression at later time points. Next, we performed a gain-of-function experiment to assess the expression of BNIP3 splice variants by western blot. Shown in Fig. 3d, expression of HIF1 $\alpha$ induced the expression of BNIP3-FL; however, when HIF1 $\alpha$ expression was combined with NF- $\mathrm{KB}$ P65, BNIP3 $\Delta$ Exon2 expression was increased and BNIP3-FL expression was proportionally decreased.

To evaluate the functional consequences of altered BNIP3 splicing, we transfected cells with HIF1 $\alpha$ and NF$\kappa \mathrm{B} P 65$, and assessed cell viability by using calcein-AM, which stains viable cells green, and ethidium homodimer1 , which stains necrotic cells red. HIF1 $\alpha$ expression increased the percentage of ethidium positive (necrotic) cells, whereas the combination of both HIF1 $\alpha$ and NF- $\mathrm{kB}$ P65 had no effect on cell death (Fig. 3e). Next, we treated HCT-116 cells with $\mathrm{CoCl}_{2}$, to induce a HIF/BNIP3 response, with or without the addition of misoprostol. Overnight treatment of cells with $\mathrm{CoCl}_{2}$ significantly increased the percentage of ethidium positive cells (Fig. 3f, g). However, when cells were treated with both $\mathrm{CoCl}_{2}$ and misoprostol, the percentage of ethidium positive cells was significantly reduced. To evaluate the role of BNIP3-FL in $\mathrm{CoCl}_{2}$-induced cell death, we performed BNIP3-FL knockdown studies. Prior to functional assays, we transfected HCT-116 cells with si-BNIP3-FL and treated with $\mathrm{CoCl}_{2}$. We observed that the siRNA reduced BNIP3-FL expression without affecting BNIP3 $\triangle$ Exon2 expression (Fig. 3h). We then assessed the functional consequences of this knockdown, by demonstrating that si-BNIP3-FL is sufficient to prevent $\mathrm{CoCl}_{2}$-induced necrotic cell death (Fig. 3i). Furthermore, in parallel gain-of-function experiments, we transfected cells with Bnip3-FL to induce necrosis, and added either Bnip $3 \Delta$ Exon3 or BNIP3 $\triangle$ Exon2. Shown in Fig. 3j, k, Bnip3-FL increased the percentage of ethidium positive cells, which was reduced by co-expression of either Bnip $3 \Delta$ Exon3 or BNIP3 $\triangle$ Exon2. These findings suggest that both Bnip3$\triangle$ Exon3 and BNIP3 $\Delta$ Exon2 act as endogenous inhibitors of Bnip3-FL-induced cell death.

\section{Misoprostol and Bnip3 $\Delta$ Exon3 protect against hypoxia- induced mitochondrial dysfunction}

Clinical evidence suggests that cardiac dysfunction, and the presence of serum cardiac marker-proteins, are important predictors of morbidity and mortality during neonatal hypoxia/asphyxia, ${ }^{5,6}$ Furthermore, recent experimental evidence has implicated mitochondrial permeability transition, rapid dissipation of the mitochondrial membrane potential, and mitochondrial superoxide production as pre-cursor events leading to regulated necrosis ${ }^{29,30}$. Thus, we evaluated the effect of misoprostol and Bnip3 $\Delta$ Exon3 on hypoxia-induced mitochondrial dysfunction in primary neonatal ventricular myocytes. First, we assessed mitochondrial membrane potential by TMRM staining. Shown in Fig. 4a, b, exposure to hypoxia $\left(10 \% \mathrm{O}_{2}\right.$ for $\left.48-\mathrm{h}\right)$ reduced mitochondrial membrane potential, which was completely attenuated by treatment with misoprostol. Next, we evaluated mitochondrial super oxide levels with MitoSOX, and observed that hypoxia-induced super oxide accumulation was prevented by misoprostol treatment (Fig. 4c, d). We also evaluated mitochondrial respiration in primary ventricular myocytes using a metabolic flux analyzer (Seahorse, XF24). Consistent with our TMRM observations, 48-h of hypoxia exposure reduced both basal and maximal respiration, which was completely abrogated by misoprostol treatment (Fig. 4e, f). Finally, we generated a Bnip3 $\Delta$ Exon3 lentivirus and evaluated if this Bnip3 splice variant could overcome mitochondrial perturbations induced by hypoxia exposure. We found that primary myocytes transduced with Bnip $3 \Delta$ Exon3 lentivirus gained resistance to mitochondrial depolarization caused by hypoxia (Fig. 4g, h). Collectively, these findings demonstrate that both misoprostol and Bnip3$\Delta$ Exon3 confer some degree of protection against 


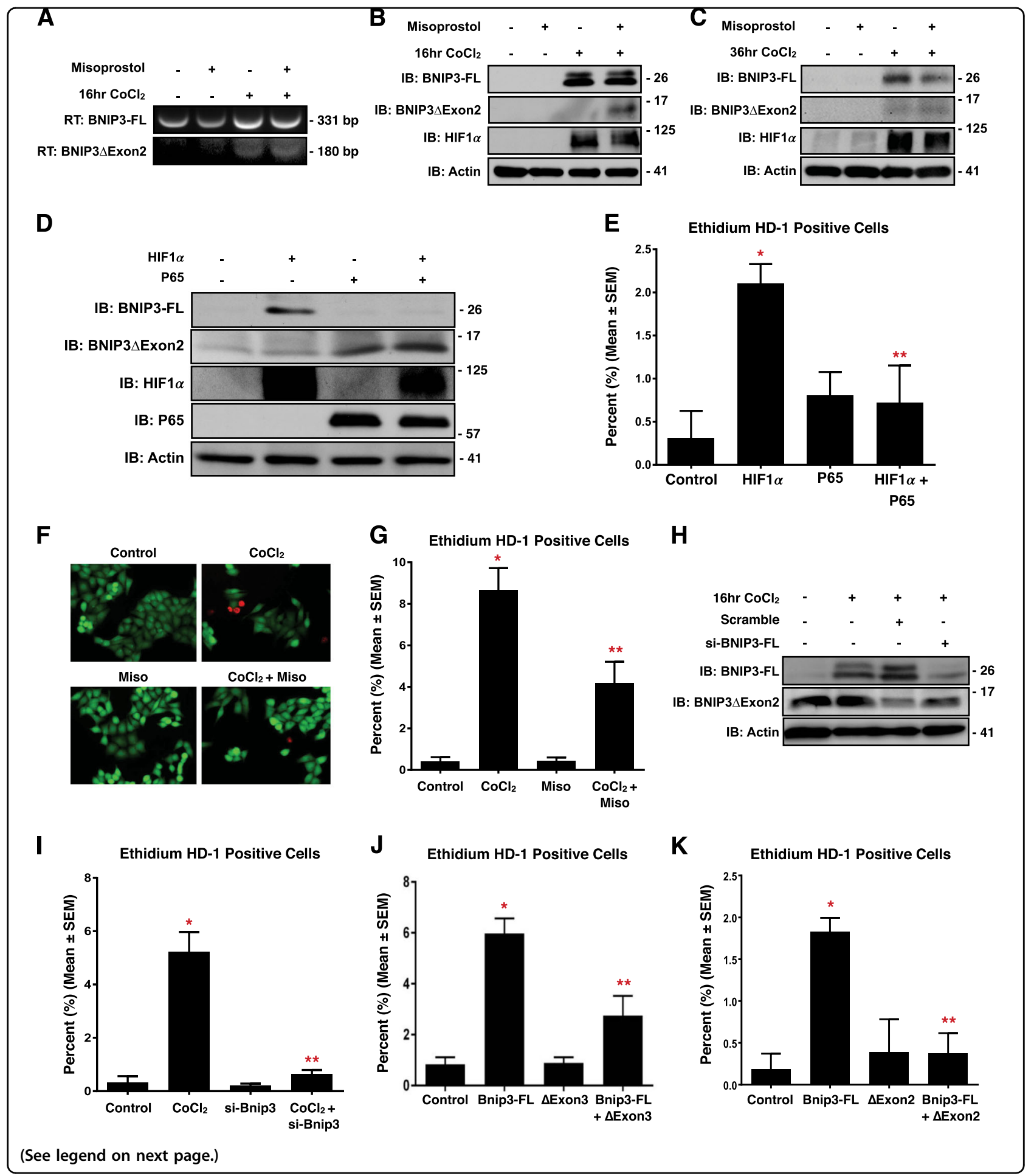

hypoxia-induced mitochondrial dysfunction in primary neonatal cardiomyocytes.

Bnip3-FL-induced mitochondrial perturbations are inhibited by the small Bnip3 splice variants

Next, we evaluated how misoprostol and Bnip3-FL affect mitochondrial membrane potential in human
(HCT-116) and rodent (H9c2) cell lines. Staining with TMRM, we observed that $\mathrm{CoCl}_{2}$ significantly reduced mitochondrial membrane potential; however, cotreatment with misoprostol completely abolished this effect (Fig. 5a, b). We assessed mitochondrial membrane potential in cultured $\mathrm{H} 9 \mathrm{c} 2$ cells, where CMV-GFP was used to identify transfected cells. Shown in Fig. 5c, 
(see figure on previous page)

Fig. 3. HIF1a and P65 drive expression of pro-survival BNIP3 splice variants. a HCT-116 cells were treated with $200 \mu \mathrm{M}$ cobalt chloride $\pm 10 \mu \mathrm{M}$ misoprostol or vehicle control for $20 \mathrm{~h}$. RNA was isolated and RT-PCR was performed for BNIP3 isoforms. $\mathbf{b}$ HCT-116 cells were treated as in $\mathbf{a}$. Protein extracts were immunoblotted, as indicated. $\mathbf{c}$ Immunoblot of protein extracts from HCT-116 cells treated with misoprostol and $\mathrm{CoCl}_{2}$ in for $36 \mathrm{~h}$. d HCT-116 cells were transfected with HIF1a \pm P65. Protein extracts were immunoblotted, as indicated. e Quantification calcein-AM and ethidium homodimer-1 stained HCT-116 cells transfected with HIF1a and/or P65. f HCT-116 cells treated with $200 \mu \mathrm{M}$ cobalt chloride $\pm 10 \mu \mathrm{M}$ misoprostol or vehicle control for $20 \mathrm{~h}$. Live cells were stained were stained with calcein-AM (green)and necrotic cells were stained with ethidium homodimer-1 (red), cells were imaged by standard fluorescence microscopy. $\mathbf{g}$ Fluorescent images were quantified by calculating the percent of necrotic cells (ethidium homodimer-1 positive) cells in 10 random fields. $\mathbf{h}$ Immunoblot of HCT-116 cells transfected with si-BNIP3-FL or scrambled control. Cells were treated with $200 \mu \mathrm{M}$ cobalt chloride or vehicle control for $20 \mathrm{~h}$. $\mathbf{i}$ HCT-116 cells treated as in $\mathbf{h}$ and cells were stained as in $\mathbf{f}$, and quantified as indicated in $\mathbf{g}$. j Quantification of HCT-116 cells transfected with Bnip3-FL, Bnip3 $\triangle$ Exon3 or empty vector control. Cells were stained, and quantified as indicated in $\mathbf{f}$. $\mathbf{k}$ Quantification of HCT-116 cells transfected with Bnip3-FL, BNIP3 $\triangle E x o n 2$ or an empty vector control. Cells were stained, and quantified as indicated in $\mathbf{f}$. Data are represented as mean \pm S.E.M. ${ }^{*} P<0.05$ compared with control, while ${ }^{* *} P<0.05$ compared with treatment, determined by 1-way ANOVA

knockdown of Bnip3 $\Delta$ Exon3 reduced the effectiveness of misoprostol in moderating the depolarization caused by treatment with $\mathrm{CoCl}_{2}$. Therefore, we decided to directly examine the effects of the smaller splice variants on membrane potential in transfection experiments. Bnip3FL expression reduced TMRM staining, where coexpression of Bnip3 $\Delta$ Exon3 and Bnip3-FL restored TMRM staining to levels comparable to control (Fig. 5d), and BNIP3 $\triangle$ Exon2 had the same effect (Fig. 5e, f). Next, we assessed mitochondrial permeability transition by staining H9c2 cells with calcein-AM, where cobalt chloride is used to quench the cytosolic emission ${ }^{31}$. With this technique, loss of mitochondrial puncta is interpreted as permeability transition. A CMV-dsRed plasmid was included to identify transfected cells. Expression of Bnip3$\mathrm{FL}$ in $\mathrm{H} 9 \mathrm{c} 2$ cells resulted in a significant decrease in the number of transfected cells with observable puncta; whereas, co-expression of either Bnip3 $\Delta$ Exon3 (Fig. 5g, h) or BNIP3 $\Delta$ Exon2 (Fig. 5i) restored the number of punctate cells. Finally, to evaluate whether these mitochondrial perturbations translated into an effect on cell viability, we transfected H9c2 cells with Bnip3-FL and with and without Bnip3 $\Delta$ Exon3, then assessed the percentage of ethidium homodimer positive cells. As shown in Fig. 5j, k, Bnip3-FL-induced cell death was reversed in the presence of Bnip3 $\Delta$ Exon3 in this cardiac cell line.

\section{Bnip3 splice variants regulate mitochondrial calcium homeostasis}

An important component of regulated necrosis involves permeability transition triggered by elevations in mito-


endoplasmic reticulum can serve as a trigger for permeability transition ${ }^{34,35}$. To more fully investigate the role of cellular calcium in Bnip3-regulated permeability transition, we used organelle-targeted plasmid-based calcium biosensors, called GECOs (Genetically Encoded $\mathrm{Ca}^{2+}$ indicators for Optical imaging) ${ }^{36}$. We used the mitochondrial matrix-targeted red GECO, known as mitocarmine (mito-Car-GECO) and ER-targeted LAR-GECO
(ER-LAR-GECO) ${ }^{37}$ to test the hypothesis that the effects of Bnip3 splice variants on mitochondrial function and cell viability are interconnected with calcium signaling. Shown in Fig. 6a, b, expression of Bnip3-FL reduced steady-state ER calcium levels. Surprisingly, Bnip3 $\Delta$ Exon3 also reduced ER calcium content and no cumulative effect on calcium release was observed with co-expression of both splice variants. Next, we examined if mitochondria were the destination of the released calcium using the mitochondrial-targeted GECO (Fig. 6c, d). We observed that Bnip3-FL increased mitochondrial calcium levels. Whereas Bnip3 $\Delta$ Exon3 had no effect when acting alone, but when together with Bnip3-FL, Bnip3 $\Delta$ Exon3 attenuated mitochondrial calcium accumulation. A similar phenomenon was observed with BNIP3 $\Delta$ Exon2 (Fig. 6e). Likewise, misoprostol had a similar effect on mitochondrial calcium accumulation when co-treating with $\mathrm{CoCl}_{2}$, to induce Bnip3-FL expression (Fig. 6f). Moreover, knockdown of Bnip3-FL with an siRNA prevented $\mathrm{CoCl}_{2}$ induced mitochondrial calcium accumulation (Fig. 6g). In concert with our earlier observations, knockdown of Bnip3 $\Delta$ Exon3 reduced the effectiveness of misoprostol in moderating the mitochondrial calcium load (Fig. 6h). These observations led us to hypothesize that Bnip3$\Delta$ Exon3 attenuates mitochondrial permeability transition by blocking mitochondrial calcium uptake, but not the release of calcium from the ER. To test this hypothesis, we expressed Bnip3-FL, with and without the inositol triphosphate receptor (IP3R) inhibitor 2-APB, and evaluated mitochondrial calcium levels. Shown in Fig. 6i, Bnip3-FLinduced mitochondrial calcium accumulation was attenuated by $2-\mathrm{APB}$, suggesting that the ER calcium release is buffered by the mitochondria. Next, we evaluated whether pharmacological inhibition of the mitochondrial calcium uniporter (MCU) with Ru360, or inhibition of the mitochondrial voltage-dependent anion channel (VDAC) with DIDS, could affect Bnip3-FL-induced mitochondrial calcium accumulation. We observed that both Ru360 and DIDS prevented mitochondrial calcium accumulation caused by Bnip3-FL (Fig. 6j). Interestingly, a combination 
A
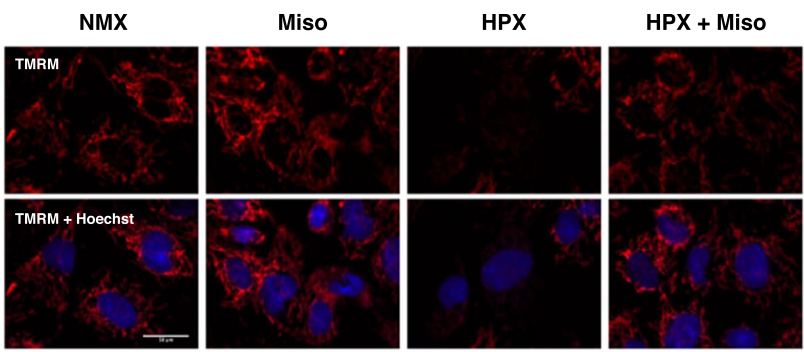

B

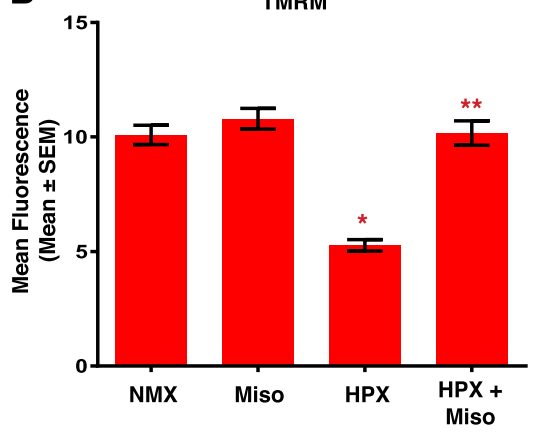

C
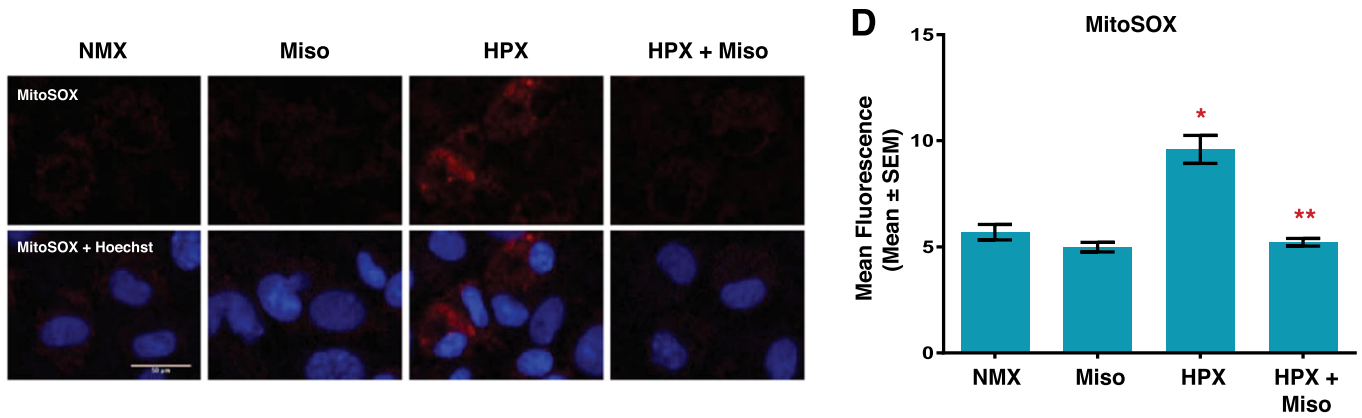


G

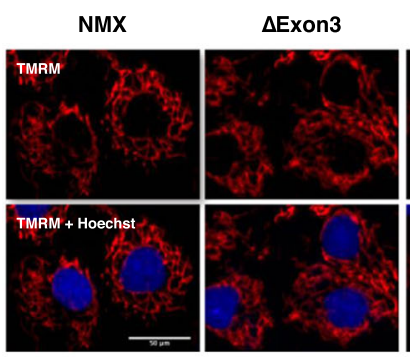

HPX

$H P X+\Delta$ Exon 3

H TMRM
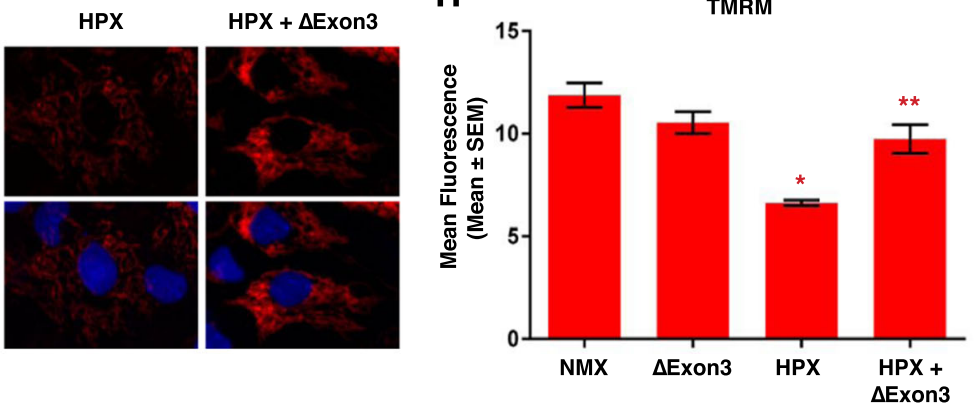

Fig. 4 (See legend on next page.)

of both inhibitors did not produce any additional effect, and that levels of mitochondrial calcium when treated with the inhibitors was similar in magnitude to that observed with co-expression of Bnip3 $\Delta$ Exon3. Finally, we used the SERCA inhibitor thapsigargin (Thaps) to circumvent a regulated ER calcium release. Shown in Fig. 6k, 
(see figure on previous page)

Fig. 4 Misoprostol opposes hypoxia-induced mitochondrial dysfunction in primary ventricular neonatal cardiomyocytes. a Primary

ventricular neonatal cardiomyocytes (PVNM) cells were treated with $10 \mu \mathrm{M}$ misoprostol (Miso) $\pm 10 \% \mathrm{O}_{2}(\mathrm{HPX})$ for $48 \mathrm{~h}$. Cells were stained with TMRM (red) and Hoechst (blue) and imaged by standard fluorescence microscopy. $\mathbf{b}$ Quantification of TMRM in $\mathbf{a}$, red fluorescent signal was normalized to cell area and quantified in 10 random fields. c PVNM cells were treated as described in a. Cells were stained with MitoSOX (red) to evaluate mitochondrial superoxides and Hoechst (blue) and imaged by standard fluorescence microscopy. d Quantification of (c), red fluorescent signal was normalized to cell area and quantified in 10 random fields. e Oxygen consumption rate (OCR) was evaluated on a Seahorse XF-24 in PVNM. To evaluate mitochondrial function, wells were injected with oligomycin $(1 \mu \mathrm{M})(\mathbf{a}), \mathrm{FCCP}(1 \mu \mathrm{M})(\mathbf{b})$, and antimycin $\mathrm{A}(1 \mu \mathrm{M})$ and rotenone $(1 \mu \mathrm{M})$ (c). $\mathbf{f}$ Calculated respiration rates from (e). $\mathbf{g}$ PVNM cells were transduced with Bnip3 $\triangle$ Exon3 $\pm 10 \% \mathrm{O}_{2}(\mathrm{HPX})$ for $48 \mathrm{~h}$. Cells were stained with TMRM (red) and Hoechst (blue) and imaged by standard fluorescence microscopy. $\mathbf{h}$ Quantification of $\mathbf{g}$, red fluorescent signal was normalized to cell area and quantified in 10 random fields. Data are represented as mean \pm S.E.M. ${ }^{*} P<0.05$ compared with control, while ${ }^{* *} P<0.05$ compared with hypoxia treatment, determined by 1-way ANOVA

thapsigargin treatment led to mitochondrial calcium accumulation that was attenuated by expression of Bnip3 $\Delta$ Exon3, indicating that Bnip3 $\Delta$ Exon3 affects the entry of calcium into the mitochondria. Collectively, these observations support our hypothesis that Bnip3-FL promotes ER calcium release that accumulates in the mitochondrial matrix, while Bnip3 $\Delta$ Exon3 and BNIP3 $\Delta$ Exon2 serve to block mitochondrial calcium accumulation.

Bnip3 was originally discovered as an interacting partner of the pro-survival protein $\mathrm{BCL}-2^{38}$. Interestingly, BCL-2 has been implicated as an important regulator of cellular calcium signaling through multiple mechanisms, including blocking mitochondrial calcium uptake through inhibition of VDAC channels ${ }^{35,39}$. Thus, we investigated the possibility of BCL-2 involvement in Bnip3 $\triangle$ Exon3regulated calcium signaling. Specifically, we tested if Bnip3 $\Delta$ Exon3 could alter the cellular location of BCL-2. We expressed Flag-tagged BCL-2, with and without HAtagged Bnip3 $\Delta$ Exon3, and performed mitochondrial and cytosolic fractionation. Western blot analysis of these fractions revealed that Bnip3 $\triangle \mathrm{Exon} 3$ reduced the cytosolic levels of BCL-2 and increased the mitochondrial BCL-2 levels, without impacting the whole-cell expression of BCL-2 (Fig. 7a). These findings suggest that Bnip3$\triangle$ Exon3 promotes mitochondrial translocation of BCL-2. Additionally, we observed that both BCL-2 and the structurally related protein MCL-1, were capable of blocking Bnip3-FL-induced mitochondrial calcium accumulation (Fig. 7b).

Our observation that Bnip3 $\Delta$ Exon3 did not block ER calcium release, but did prevent mitochondrial calcium accumulation, led us to investigate the nucleus as a possible subcellular destination for this calcium. Using a GECO-based calcium biosensor fused to a nuclear localization signal (NLS-R-GECO) ${ }^{36}$, we evaluated the effect of Bnip3 $\Delta$ Exon3 on nuclear calcium content. Shown in Fig. 7c, d, Bnip3 $\Delta$ Exon3 increased nuclear calcium levels, whereas Bnip3-FL had no effect. Although not as marked of an elevation as observed with Bnip3 $\Delta$ Exon3 alone, nuclear calcium remained elevated when Bnip3-FL was co-expressed with Bnip3 $\Delta$ Exon3. Similar effects on nuclear calcium were observed with BNIP3 $\Delta$ Exon2 (Fig. 7e, f). In addition, we observed that Bnip3 $\Delta$ Exon3induced calcium accumulation was prevented by the IP3R inhibitor 2-APB (Fig. 7g, h), providing evidence for a dependence on ER-calcium as the source of nuclear calcium. Once again, we used thapsigargin to circumvent a regulated ER calcium release and observed no additional effect on Bnip3 $\Delta$ Exon3-induced nuclear calcium accumulation (Fig. 7i). Finally, we examined the interconnectivity of misoprostol and Bnip3 $\Delta$ Exon3 in this signaling pathway. We found that treatment with misoprostol also increased nuclear calcium levels. Importantly, this effect was diminished by siRNA knockdown of Bnip3 $\Delta$ Exon3 (Fig. 7j, k). Collectively, these observations provide evidence that Bnip3 $\Delta$ Exon3 promotes ER-tonuclear calcium transfer, while Bnip3-FL promotes ER-tomitochondrial calcium transfer. Furthermore, mitochondrial translocation of BCL-2 might be an important intermediate step in the distinct regulation of calcium signals initiated by Bnip3 $\Delta$ Exon3.

\section{Bnip3 $\Delta$ Exon3 promotes cardiomyocyte hypertrophy}

In order to understand the cellular consequences of Bnip3 $\Delta$ Exon3-induced nuclear calcium accumulation, we evaluated two transcriptional regulators known to be regulated in a calcium-dependent manner. Theoretically, these regulators (NFATc3 and HDAC5) offer a tool to test the potential of Bnip3 $\Delta$ Exon3-regulated calcium signaling as a modulator of gene expression; biologically, these tools serve as indications of the cellular significance of this regulatory pathway. First, the transcription factor NFATc3 is activated by the calcium-calmodulin activated phosphatase calcineurin, promoting nuclear translocation. Second, the class II histone deacetylase, HDAC5, is exported from the nucleus upon phosphorylation by calcium-calmodulin activated kinases (CaMKs). Thus, we evaluated the effect of Bnip3 $\Delta$ Exon3 on the subcellular localization of these proteins as an indication of their activity by way of fluorescent fusion proteins of NFATc3 (NFAT-YFP) and HDAC5 (HDAC5-GFP). We observed 





(see figure on previous page)

Fig. 5 Bnip3 splice variants oppose mitochondrial perturbations. a HCT-116 cells treated with $200 \mu \mathrm{M}$ cobalt chloride $\pm 10 \mu \mathrm{M}$ misoprostol or vehicle control for $20 \mathrm{~h}$. Cells were stained with TMRM (red) and Hoechst (blue) and imaged by standard fluorescence microscopy. b Quantification of TMRM in a, red fluorescent signal was normalized to cell area and quantified in 10 random fields. c Quantification of H9c2 cells treated with $200 \mu \mathrm{M}$ cobalt chloride $\pm 10 \mu \mathrm{M}$ misoprostol or vehicle control for $20 \mathrm{~h}$, and transfected with si-Bnip3 $\Delta$ Exon3 or a scrambled control. Cells were stained as in a and quantified as in $\mathbf{b}$. d Quantification of H9c2 cells was transfected with Bnip3-FL, Bnip3 Exon3, or an empty vector control. TMRM staining was quantified as in b. e H9c2 cells were transfected with Bnip3-FL, BNIP3 $\triangle E x o n 2$, or empty vector control. Outlines indicate CMV-GFP positive cells, included to identify transfected cells. Cells were stained as in $\mathbf{a}$. $\mathbf{f}$ Quantification of (e), red fluorescent signal was normalized to cell area and quantified in 10 random fields. $\mathbf{g} \mathrm{H} 9 \mathrm{c} 2$ cells were transfected with Bnip3-FL, Bnip3 $\triangle$ Exon3, or empty vector control. CMV-dsRed (red) was used to identify transfected cells. Cells were stained with calcein-AM and cobalt chloride $\left(\mathrm{CoCl}_{2}, 5 \mu \mathrm{M}\right)$ to assess permeability transition. $\mathbf{h}$ Quantification of $\mathbf{g}$ by calculating the percentage of cells with punctate calcein signal in 10 random fields. i Quantification of H9c2 cells transfected with Bnip3-FL, BNIP3 Exon2, or empty vector control. CMV-dsRed was used to identify transfected cells. Cells were stained and quantified as indicated in $\mathbf{h}$. $\mathbf{j} H 9 c 2$ cells transfected with Bnip3-FL, BNIP3 $\triangle$ Exon3, or empty vector control. Live cells were stained with calcein-AM (green), and necrotic cells were stained with ethidium homodimer-1 (red), cells were imaged by standard fluorescence microscopy. $\mathbf{k}$ Fluorescent images in $\mathbf{j}$ were quantified by calculating the percent of necrotic cells (ethidium homodimer-1 positive) cells in 10 random fields. Data are represented as mean \pm S.E.M. ${ }^{*} P<0.05$ compared with control, while ${ }^{* *} P<0.05$ compared with Bnip3-FL treatment, determined by 1-way ANOVA

that expression of Bnip3 $\Delta$ Exon3 increased the nuclear localization of NFAT-YFP (Fig. 8a, b), and increased the cytosolic localization of HDAC5-GFP (Fig. 8c, d). These observations suggest that Bnip3 $\Delta$ Exon3-induced nuclear calcium has the potential to alter gene expression. Interestingly, both NFATs and class II HDACs have been implicated as regulators of cardiomyocyte growth during development and cardiac disease. Thus, as a test of the cellular significance of this regulatory axis we transduced primary neonatal cardiomyocytes with a lentivirus delivering Bnip3 $\Delta$ Exon3 and performed immunofluorescence with the sarcomeric myosin antibody MF-20 to measure cell area. Shown in Fig. 8e, f, we observed a statistically significant increase in cardiomyocyte cell area with Bnip3 $\Delta$ Exon3 expression. Furthermore, we evaluated the expression of two classical marker genes of cardiac hypertrophy and reactivation of the "fetal gene program", $\alpha$ - and $\beta$-myosin heavy chain (MHC), by real-time PCR. In this regard, a shift from $\alpha$-MHC (Myh6) to $\beta$-MHC $(M y h 7)$ serves as an indication of the reversion from "adult" to "fetal" gene expression programs ${ }^{40,41}$. We observed that Bnip3 $\Delta$ Exon3 increased Myh7 expression and reduced Myh6 expression (Fig. 8g). This resulted in an increase in the MHC ratio $(M y h 7 / M y h 6 ; 1.66)$ with Bnip3 $\Delta$ Exon3 transduction. In addition, Bnip3 $\Delta$ Exon3 transduction increased the expression of atrial natriuretic factor (ANF) in primary cardiomyocytes (Fig. 8h). Similarly, we observed that treatment of primary cardiomyocytes with misoprostol also increased cell area, and the magnitude of this effect was matched in cells treated with the well-established hypertrophy-inducer phenylephrine (PE) (Fig. 8i). Collectively, these findings suggest that nuclear accumulation of calcium elicited by the small Bnip3 splice variants operates to regulate the activity of transcription regulators and has the potential to control cardiomyocyte growth, in addition to their roles in cell survival.

\section{Discussion}

The cellular response to hypoxia involves both adaptive cell survival phenomenon and regulated cell death; however, the mechanisms dictating these opposing cell phenotypes during development and disease remain poorly defined. Here, we provide evidence that activation of prostaglandin signaling through misoprostol treatment induces pro-survival NF- $\mathrm{BB}$ gene expression during hypoxia, resulting in reduced expression of the pro-death Bnip3-FL splice variant and increased expression of prosurvival small Bnip3 variants. Our data also implicates the combined action of HIF1 $\alpha$ and NF- $\kappa B$ P65 to promote cell survival, whereas activation of HIF1 $\alpha$ alone induces Bnip3-FL expression and cell death. We also demonstrate that the BNIP3 gene can be differentially spliced in human versus rodent cells.

Since its discovery, it has been noted that Bnip3 could induce context-dependent apoptotic or necrotic cell death ${ }^{14,38}$. Early studies evaluating Bnip3 determined its subcellular location is predominantly mitochondrial, and cells expressing Bnip3 displayed features consistent with apoptosis $^{38}$. However, more detailed analysis of Bnip3 revealed that it could activate cell death from extramitochondrial sites and cells expressing Bnip3 display a necrotic cell phenotype, particularly when Bnip3 is targeted to the ER with the cytochrome-b5 transmembrane domain ${ }^{12,14}$. Importantly, cell death elicited by Bnip3 is attenuated with the mitochondrial permeability transition pore inhibitor cyclosporine-A and the MCU blocker Ru360 ${ }^{12,14}$; whereas, cells expressing BNIP3 are resistant to caspases inhibitors, such as zVAD-fmk ${ }^{14}$. These findings are consistent with the notion that Bnip3 is an important regulator of mitochondrial permeability transition-regulated necrosis, through a calciumdependent mechanism ${ }^{29,30}$. Current models of Bnip3 function suggest that Bnip3 regulates apoptosis and mitophagy when localized at the mitochondria, and 


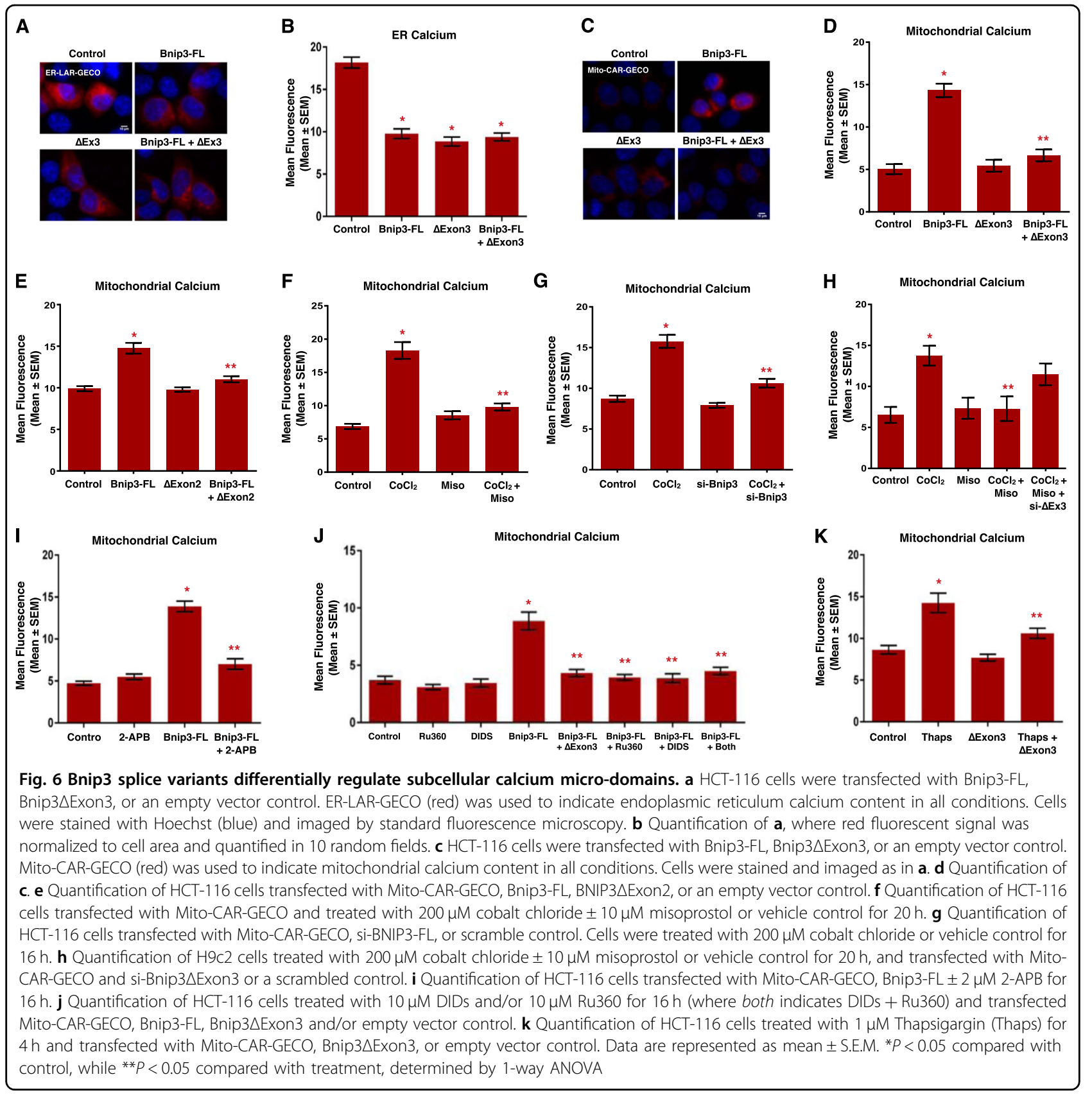

macro-autophagy and necrosis when localized at the ER membranes ${ }^{13}$. However, a definitive mechanism describing how Bnip3 is translocated to either the mitochondria or ER is lacking, and many questions remain regarding cell-type specific differences in Bnip3 function.

The results of the present study expand these previous observations and suggest that alternative splicing of Bnip3 is an important mechanism to control cellular calcium homeostasis, cell death, and cell growth. We observed that both Bnip3-FL and Bnip3 $\Delta$ Exon3 deplete ER calcium content. However, Bnip3-FL preferentially transfers calcium to the mitochondrial matrix and triggers mitochondrial permeability transition and necrosis; whereas, Bnip3 $\Delta$ Exon3 serves to block mitochondrial accumulation and promote nuclear calcium transfer leading myocyte cell growth. However, the role of the endogenous Bnip3 splice variants has not been fully validated in vivo, and the function of specific splice variants has yet to be characterized by gene-targeting studies; however, NF- $\mathrm{kB}$ activity appears to be required for the induction of the small Bnip3 splice variants and the activity of this transcription factor complex has been demonstrated during cardiac hypertrophy ${ }^{15,42}$. Our findings serve to unify the seemingly disparate observations of 
A

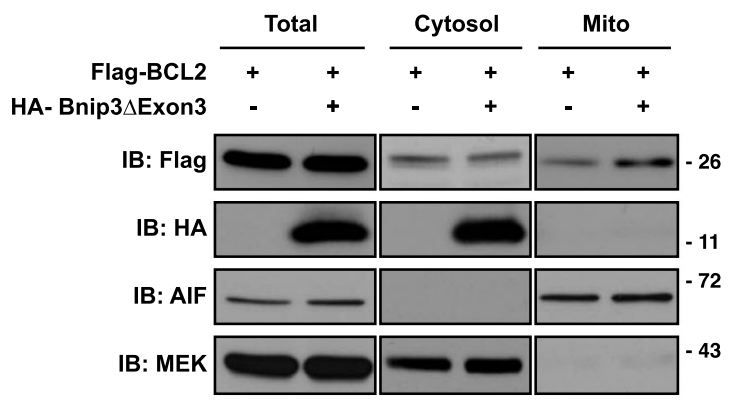

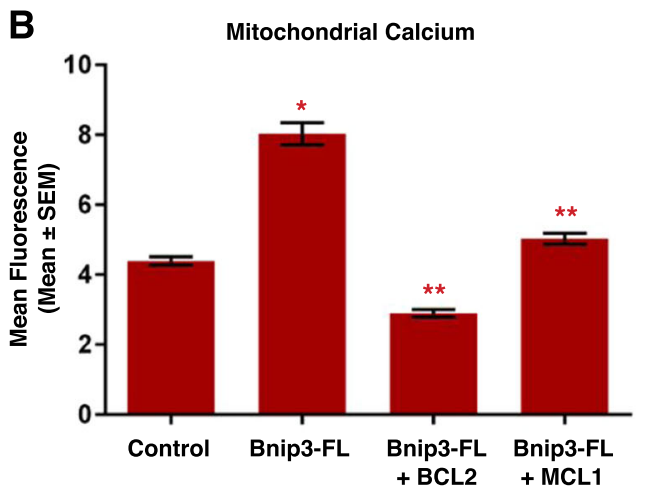

E
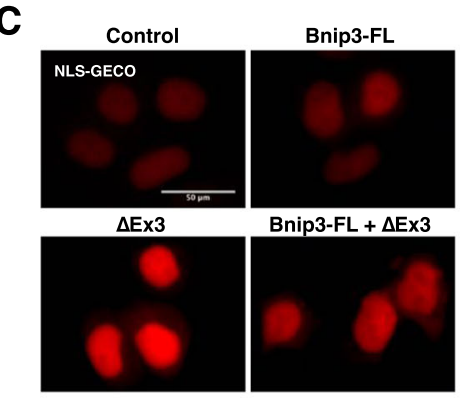

$\mathbf{F}$

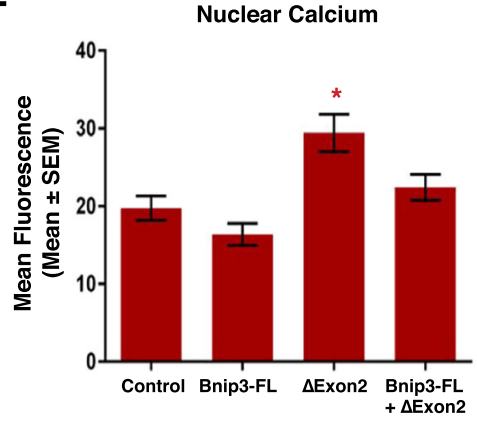

I

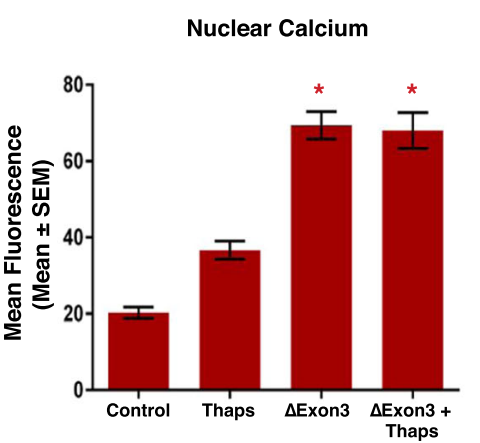

D

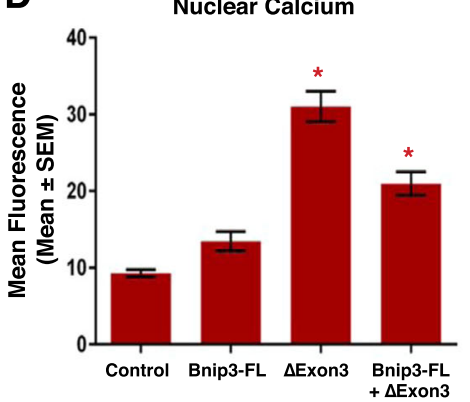

G

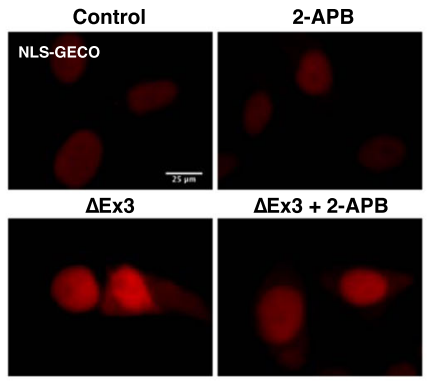

J

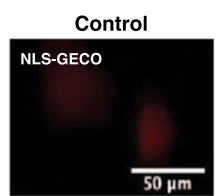

si- $\Delta \mathrm{Ex} 3$



Miso

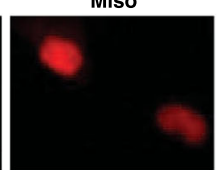

Miso+ si- $\Delta \mathrm{Ex} 3$

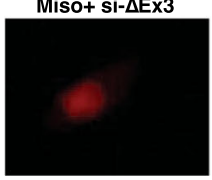

H Nuclear Calcium
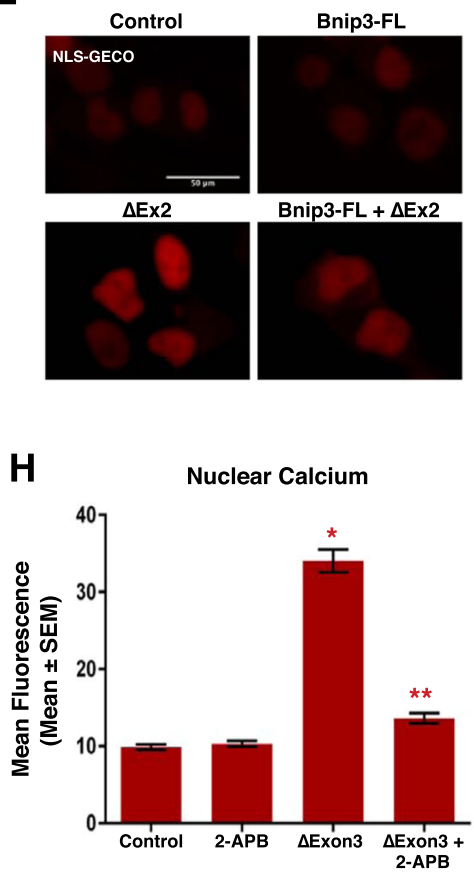

K

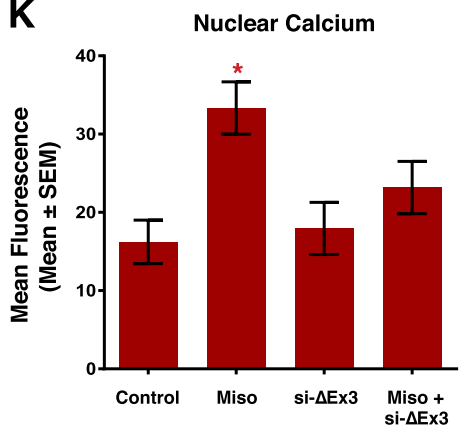

Fig. 7 (See legend on next page.) 
(see figure on previous page)

Fig. 7 Bnip3 splice variants regulate nuclear calcium content. a HCT-116 cells were transfected with Flag-BCL2 and HA-Bnip3 $\triangle E x O n 3$, as indicated. Protein extracts were subjected to fractionation and were immunoblotted, as indicated. $\mathbf{b} \mathrm{HCT}-116$ cells were transfected with Mito-CARGECO, Bnip3-FL, Flag-BCL2, or MCL-1, as indicated. Fluorescence was normalized to cell area and quantified in 10 random fields. c HCT-116 cells were transfected with Bnip3-FL, Bnip3 $\triangle$ Exon3, or an empty vector control. NLS-R-GECO (red) was used to indicate nuclear calcium content. Cells were stained with Hoechst (blue) and imaged by standard fluorescence microscopy. d Quantification of (c), where red fluorescent signal was normalized to nuclear area and quantified in 10 random fields. e HCT-116 cells were transfected with NLS-R-GECO, Bnip3-FL, BNIP3 $\triangle E x o n 2$, or an empty vector control. $\mathbf{f}$ Quantification of $\mathbf{e . ~} \mathbf{g} \mathrm{HCT}-116$ cells were transfected with NLS-R-GECO, Bnip3 $\triangle$ Exon3 $\pm 2 \mu \mathrm{M}$ 2-APB for 16 h. $\mathbf{h}$ Quantification of $\mathbf{g}$. i HCT-116 cells transfected with NLS-R-GECO, Bnip3 $\triangle$ Exon3 $\pm 1 \mu \mathrm{M}$ Thapsigargin (Thaps) for $4 \mathrm{~h}$. $\mathbf{j}$ H9c2 cells transfected NLS-R-GECO, si-Bnip3 $\Delta$ Exon3 (indicated as si- $\triangle$ Ex3) or scrambled control, and treated with $10 \mu \mathrm{M}$ misoprostol or vehicle control for $20 \mathrm{~h}$. Cells were stained with Hoechst (blue) and imaged by standard fluorescence microscopy. $\mathbf{k}$ Quantification of $\mathbf{j}$. Data are represented as mean \pm S.E.M. ${ }^{*} P<0.05$ compared with control, while ${ }^{* *} P<0.05$ compared with treatment, determined by 1-way ANOVA or unpaired t-test

others who have observed that IP3R-dependent calcium release can induce cell death ${ }^{34,35}$, and cardiac hypertrophy ${ }^{43}$, where the splicing of Bnip3, and the cellular location of BCL-2, can influence cell fate by dictating where the IP3R-dependent calcium release accumulates (i.e., mitochondria or the nucleus). However, additional in vivo experimentation is required to evaluate the role of Bnip3 splicing in hypertrophic regulation and determine under which biological contexts this Bnip3-dependent mechanism operates, as other BCL-2 family members, such as Bnip3L/Nix, likely contribute to cardiac calcium homeostasis ${ }^{37}$.

Another important observation in the present study is the identification of the human BNIP3 $\Delta$ Exon2 splice variant. Although we identified several similarities between BNIP3 $\triangle$ Exon2 and Bnip3 $\triangle$ Exon3, such as inhibition of cell death, prevention of ER-mitochondrial calcium transfer, and nuclear calcium accumulation, potential differences likely exist. In silico domain mapping revealed conserved $\mathrm{N}$-terminal and $\mathrm{C}$-terminal sequences; however, significant sequence differences exist, including a predicted nuclear localization sequence present in BNIP3 $\triangle$ Exon2 that is not present in Bnip3 $\Delta$ Exon3, and the LIR domain which is present in Bnip3 $\Delta$ Exon3 but not BNIP3 $\triangle$ Exon2. Further experimental work is required to fully define how BNIP3 $\Delta$ Exon2 and Bnip $3 \Delta$ Exon3 functions are distinct. Interestingly, recent work regarding the Bnip3 homologue Bnip3L/Nix has demonstrated that its small splice variant, named sNix (short Nix), alters gene expression to promote cell survival in an NF- $\mathrm{kB}$-dependent manner ${ }^{44}$. Furthermore, the p65 subunit of NF- $k B$ has been shown to physically interact with NFATc1 to regulate cardiac hypertrophy ${ }^{42}$. Collectively, these findings suggest an interplay between Bnip3 and Bnip3L/Nix splicing and NF-kB-dependent gene expression to promote survival and myocyte growth. Moreover, the expression ratio of Nix to sNix was determined to be approximately 10:1, which is consistent with our findings regarding ratio of Bnip3 to its small survival splice variants, and suggests that these small splice variants exert their physiological effects through gene expression amplification rather than direct inhibition of the fulllength isoforms ${ }^{44}$. Given the similarities between Bnip3 and Nix splice variants, we propose a nomenclature of short Bnip3, or "sNip", for the small pro-survival Bnip3 splice variants, to be consistent with the Nix nomenclature ${ }^{44,45}$.

Recent progress has been made regarding the mitochondrial permeability transition pore structure and function, and several studies have defined the importance of permeability transition-dependent necrosis in the pathophysiology of ischemic cardiac and cerebrovascular diseases $^{30}$. Our data suggests that Bnip3 splicing plays an important role in the complications arising from neonatal hypoxia. However, additional studies are needed to determine if this mechanism operates to control permeability transition, necrosis, and/or cell growth in other pathologies. Given the diverse roles of Bnip3 splice variants regulating mitochondrial function, autophagy, and cell growth, dysregulation of this genetic pathway may have broad implications involving ischemic cardiovascular and cerebrovascular diseases, as well as cancer biology.

\section{Materials and methods}

In vivo neonatal hypoxia model

All procedures in this study were approved by the Animal Care Committee of the University of Manitoba, which adheres to the principles for biomedical research involving animals developed by the Canadian Council on Animal Care. Litters of Long-Evans rat pups $(n=5)$ and their dam were placed into a hypoxia chamber with $10 \%$ oxygen delivery, at post-natal day-3 (PND3) for 7 days. Control pups $(n=5)$ were left in normoxic conditions at $21 \%$ oxygen $^{23,24}$. Animals received subcutaneous injections of $10 \mu \mathrm{g} / \mathrm{kg}$ misoprostol, or saline control, for 7 days. At PND10, animals were euthanized and perfused with saline for tissue collection.

\section{Plasmids and virus production}

The PKA biosensor (pPHT-PKA) was a gift from Anne Marie Quinn (Addgene \#60936) ${ }^{26}$. The plasmids for human P65 expression including: GFP-RelA, T7-RelA, 


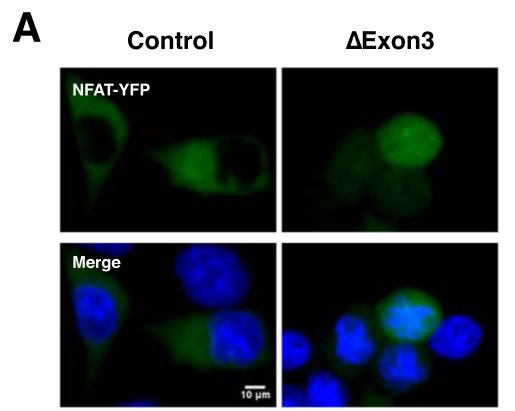

D

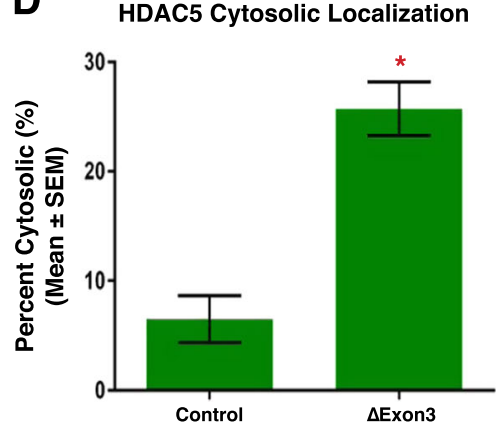

G Myosin Heavy Chain Expression

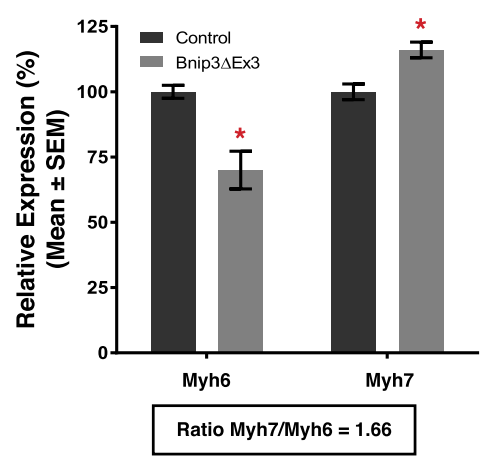



C

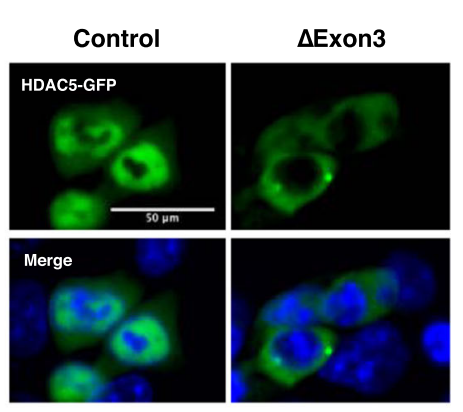

E

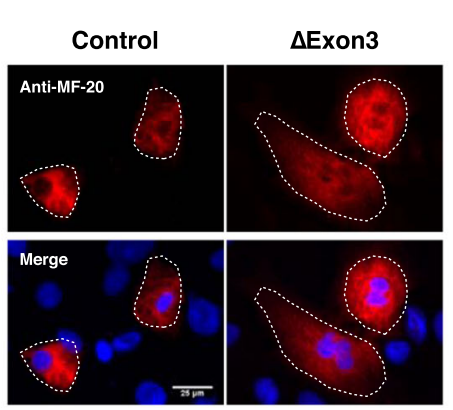

$\mathbf{F}$



H

I

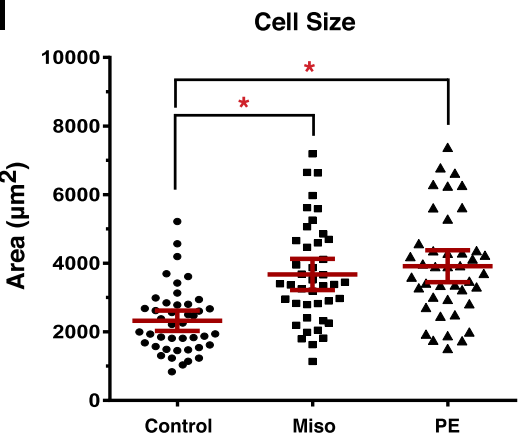

Fig. 8 Bnip3 3 Exon3 regulates cardiomyocyte hypertrophy. a HCT-116 cells were transfected with Bnip3 $\triangle$ Exon3 or an empty vector control. NFAT-YFP (green) was used to indicate subcellular localization of NFAT. Cells were stained with Hoechst (blue) and imaged by standard fluorescence microscopy. $\mathbf{b}$ Quantification of fluorescent images in $\mathbf{a}$, by calculating the percentage of cells with nuclear NFAT signal over 10 random fields. $\mathbf{c}$ HCT116 cells were transfected with Bnip3 $\triangle$ Exon3 or an empty vector control. HDAC5-GFP (green) was used to indicate subcellular localization of HDAC5. Cells were stained and imaged as in $\mathbf{a}$. $\mathbf{d}$ Quantification of fluorescent images in (c), by calculating the percentage of cells with cytosolic

HDAC5 signal over 10 random fields. e Primary ventricular neonatal cardiomyocytes (PVNM) cells were transduced with Bnip3 $\triangle$ Exon3 or control virus. Cells were fixed, stained with Hoechst (blue), and probed for myosin heavy chain (Anti-MF-20, red) expression. $\mathbf{f}$ Quantification of e, where cell size $\left(\mu \mathrm{m}^{2}\right)$ was calculated based on the area of the red fluorescent signal and quantified in 10 random fields. $\mathbf{g}, \mathbf{h}$ PVNM cells treated as in e. RNA was isolated and qRT-PCR was performed for myosin heavy chain isoform and ANF expression. i Quantification of PVNM cells treated with $10 \mu \mathrm{M}$ misoprostol, $10 \mu \mathrm{M}$ phenylephrine (PE), or vehicle control for $20 \mathrm{~h}$. Cells were stained with calcein-AM (green) and assessed for cell size. Cell size $\left(\mu \mathrm{m}^{2}\right)$ was calculated based on the area of the green fluorescent signal and quantified in 10 random fields. Data are represented as mean \pm S.E.M. ${ }^{*} P<0.05$ compared with control, determined by unpaired t-test

T7-RelA(S276A), and PCMV4-3 HA/IkB-alpha (SS32,36AA) were gifts from Warner Greene (Addgene $\# 23255,21984,24153,24143)^{46,47}$. The plasmid for HIF1 $\alpha$ (HA- HIF1 $\alpha$-pcDNA3) was a gift from William Kaelin
(Addgene \#18949) $)^{48}$. The mitochondrial (CMV-mitoCAR-GECO1), endoplasmic reticulum (CMV-ER-LARGECO1), and nuclear (CMV-NLS-R-GECO) targeted calcium biosensors were gifts from Robert Campbell 
(Addgene \#46022, 61244, and 32462) ${ }^{36,49,50}$. Flag-BCL-2 was gift from Clark Distelhorst (Addgene $\# 18003)^{51}$. pMSCV-puro-mMCL1-WT was a gift from Joseph Opferman (Addgene $\# 45817)^{52}$. NFATc3 fused to YFP (NFAT-YFP) was a gift from Tetsuaki Miyake, and HDAC5-GFP was provided by E. Olson. The mouse mycBnip3-FL and HA-Bnip3 $\Delta$ Exon3 (Accession \#MF156210) were described previously (Addgene \#100796, \#100793) 22 . The pLenti-Bnip3 $\triangle$ Exon3 virus was generated by ligating a PCR amplicon (HA- Bnip3 $\triangle$ Exon3) into the pLentipuro backbone, which was a gift from Ie-Ming Shih (Addgene \#39481) ${ }^{53}$. Virus was packaged and purified in the University of Manitoba Lentiviral Vector Viral Particles \& Production Core. Human HA-BNIP3-FL and HABNIP3 $\triangle$ Exon2 (Accession \#MF593120) (Addgene \#100781, \#100782) were generated by PCR using cDNA from HUECs and primers containing EcoRI and XhoI restriction enzymes sites in the forward and reverse primers, respectively. Primers include BNIP3 Forward: 5' TATGCGGAATTCATGTCGCAGAACGGAGCGCCCG GGAT-3', BNIP3 Reverse: 5'-AATCCGCTCGAGTC AAAAGGTGCTGGTGGAGGTTGTC-3'; BNIP3 $\triangle$ Exon2 Reverse: 5'-AATCCGCTCGAGTCAAGATGCTTTCAA CTTCTTTCC-3'. PCR products were cut and ligated into pcDNA3, in frame with an N-terminal HA tag, described previously $^{54,55}$.

\section{Cell culture, transductions, and transfections}

HCT-116 cells were maintained in McCoy's 5A medium (Hyclone), containing penicillin, streptomycin, and $10 \%$ fetal bovine serum (Hyclone), at $37^{\circ} \mathrm{C}$ and $5 \% \mathrm{CO}_{2}$. $\mathrm{H} 9 \mathrm{c} 2$ and 3T3 cells were maintained in Dulbecco's modified Eagle's medium (DMEM; Hyclone), containing penicillin, streptomycin, and $10 \%$ fetal bovine serum (Hyclone), at $37^{\circ} \mathrm{C}$ and $5 \% \mathrm{CO}_{2}$. Rat primary ventricular neonatal cardiomyocytes (PVNC) were isolated from 1-2-day-old pups using the Pierce Primary Cardiomyocyte Isolation Kit (\#88281). All cells were transfected using JetPrime Polyplus reagent, as per the manufacturer's protocol ${ }^{31}$. Overexpression of Bnip3 $\triangle$ Exon3 in PVNC cells was carried out using pLenti-puro-Bnip3 $\Delta$ Exon3 lentiviral particles, alongside control lentiviral particles (Santa Cruz, sc108080) to control for the effects of transduction alone. RNAi experiments targeting Bnip3-FL and Bnip3 $\Delta$ Exon3 were performed using siRNA targeting the 3rd exon (sense sequence: UCGCAGACACCACAAGAUA) and the exon 2-4 junction (5'-CACUGUGACAGUCUG AGGA) respectively (Dharmacon). A scrambled siRNA was used as control (Santa Cruz sc-37007). siRNAs were transfected into cells using JetPrime Polyplus reagent, as per the manufacturer's protocol ${ }^{31}$. For misoprostol treatments, $10 \mathrm{mM}$ misoprostol (Sigma) in phosphate buffered saline (PBS; Hyclone) was diluted to $10 \mu \mathrm{M}$ directly in media and applied to cells for $20 \mathrm{~h}^{22}$. For H89 treatment, a $10 \mathrm{mM}$ stock of H89-dihydrochloride hydrate (Sigma) in PBS, was diluted to $10 \mu \mathrm{M}$ directly in media. For cobalt chloride $\left(\mathrm{CoCl}_{2}\right)$ treatment, a $200 \mathrm{mM}$ stock solution of cobalt(II) chloride hexahydrate (Sigma) in PBS, was diluted to $200 \mu \mathrm{M}$ directly in media and applied to cells. $\mathrm{CoCl}_{2}$ concentration was selected based on previously published data looking at HIF1 $\alpha$ induction ${ }^{56}$. For calcium imaging experiments, $1 \mu \mathrm{M}$ Thapsigargin (Sigma) was applied directly to cells for $4 \mathrm{~h}$, while $2 \mu \mathrm{M} 2$-APB (Sigma), $10 \mu \mathrm{M}$ Ru360 (Sigma), and $10 \mu \mathrm{M}$ disodium 4,4' diisothiocyanatostilbene-2,2'-disulfonate (DIDS, Sigma) were applied directly to cells for $16 \mathrm{~h}$. For cardiac hypertrophy experiments, a $10 \mathrm{mM}$ stock of phenylephrine (Sigma) in PBS, was diluted to $10 \mu \mathrm{M}$ directly in media.

\section{Fluorescent staining and live cell imaging}

Hoechst 33342, TMRM, calcein-AM, and Ethidium Homodimer-1 were all purchased from Biotium and applied using manufacturer's protocol ${ }^{31,57}$. MitoSOX was purchased from Life Technologies and was applied to cells for a 10-min incubation to assess mitochondrial superoxide $^{31}$. Calcein-Cobalt Chloride $\left(\mathrm{CoCl}_{2}\right)$ staining was used to assess mitochondrial permeability transition, which is achieved through quenching cytosolic calcein$\mathrm{AM}$ signal with $5 \mu \mathrm{M} \mathrm{CoCl}_{2}$ described previously ${ }^{31}$. Immunofluorescence with Anti-MF-20 (DSHB \# $\left.A B \_2147781\right)$ was used with fluorescent secondary antibody conjugated to Alexa Fluor 555 (Themo \# A-31570) to assess cardiac hypertrophy in fixed and permeabilized PVNM cells. All imaging experiments were done on an Olympus IX70 inverted microscope with QImaging Retiga SRV Fast 1394 camera using NIS Elements AR 3.0 software. Quantification, scale bars, and processing including background subtraction, was performed on Fiji (ImageJ) software.

\section{Immunoblotting}

Protein extractions were achieved using a RIPA lysis buffer combined with phenylmethanesulfonyl fluoride (PMSF, Sigma), sodium orthovanadate (NaVO, Sigma), and phosphatase inhibitor cocktail (PIC, Santa Cruz). Homogenization was required in the case of tissue protein extraction. Mitochondrial/cytosolic fractionation was done using a Mitochondrial Isolation Kit (Qiagen Qproteome \# 37612), while nuclear/cytosolic fractionation was done using a NE-PER Nuclear and Cytosolic Extraction Kit (Pierce \# 78833), as described previously ${ }^{21,58,59}$. Protein content was determined using a Bio-Rad Protein Assay Kit. Extracts were resolved via SDS-PAGE and later transferred to a PVDF membrane using an overnight transfer system. Immunoblotting was carried out using primary antibodies in $5 \%$ powdered milk or BSA (as per manufacturer's instructions) dissolved in TBST. Horseradish peroxidase-conjugated secondary antibodies 
(Jackson ImmunoResearch Laboratories; 1:5000) were used in combination with enhanced chemiluminescence (ECL) to visualize bands ${ }^{58,59}$. The following antibodies were used: Bnip3-FL and BNIP3 $\triangle$ Exon2 (CST \# 44060), P65 (CST \# 8242), P65 Phospho-Ser276 (Assay BioTech \# A7169), HIF1 $\alpha$ (CST \# 14179), Flag (CST \# 2368), HA (CST \# 3724), AIF (CST \# 5318), MEK (CST \# 8727), Histone-H3 (CST \# 4499), Tubulin (CST \# 86298), and Actin (Santa Cruz sc-1616). For the detection of rodent Bnip3 splice variants a custom rabbit polyclonal antibody was generated by Abgent using the following peptide sequence CSQSGEENLQGSWVE.

\section{Mitochondrial respiration}

Mitochondrial respiration in PVNM cells was assessed using the Seahorse XF-24 Extracellular Flux Analyzer (Seahorse Bioscience, North Billerica, MA, USA), as described previously ${ }^{31}$. Calculated respiration rates were determined as per manufacturer's instructions (Mito Stress Kit; Seahorse Bioscience).

\section{Reverse transcription PCR}

Total RNA was extracted from cells and pulverized tissues by the TRIzol extraction method and genomic DNA was removed via the RNeasy Mini Kit (Qiagen), including an On-Colum DNase I Digestion (Qiagen). For reverse transcription PCR (RT-PCR), purified mRNA was reverse transcribed using Maxima Enzyme (Thermo), followed by PCR using Taq polymerase (New England Biolabs). Amplified RNA was then run on a $2 \%$ agarose gel with GelRed nucleic acid gel stain (Biotium). The cloning primers, described above, were used to detect human BNIP3 splice variants. Mouse Bnip3 primers were: Forward: 5'-GCCGGAATTCATGTCGCAGAGCGGGGA G-3', Bnip3-FL Reverse: 5'-CGGCGCTCGAGTCAG AAGGTGCTAGTGGAAGTTGTC-3', and Bnip $3 \triangle$ Exon3 Reverse: 5'-CGGCGCTCGAGTCAGGATACTTTCAA CTTCTCTTCTTCTCTC-3'.

Primers used for rat heart tissue were: 5'TTCCAGCTTCCGTCTCTATTT-3' and 5'-TCAGGAT ACTTTCAACTTCTCTTCT-3'. For quantitative realtime PCR (qPCR) targeting myosin heavy chain expression, mRNA was extracted from cells using TRIzol then reverse transcribed into cDNA. Following DNase treatment, cDNA was combined with SYBR Green Supermix (Thermo) and mRNA was amplified using the ABI 7500 Real-Time PCR system (Applied Biosystems). Primers were: Rat myosin heavy chain 6 forward $5^{\prime}$-GAGGAAT AACCTGTCCAGCAG - $3^{\prime}$, and reverse 5'-TACAGGC AAAGTCAAGCATTC-3'. Rat myosin heavy chain 7 forward 5'-CCAACACCAACCTGTCCAAG-3', and reverse 5'-CAAAGGCTCCAGGTCTCAGG-3'. Rat betaactin forward 5'-CTGTGTGGATTGGTGGCTCTA-3', and reverse 5' ${ }^{\prime}$-AAAACGCAGCTCAGTAACAGTCC-3'.
Rat ANF forward 5'-GGTAGGATTGACAGGATTGG A-3', and reverse 5'-GAGCAGATTTGGCTGTTATCT TC-3'.

\section{Statistics}

Data are presented as mean \pm standard error of the mean (S.E.M.). Differences between groups in imaging experiments with only 2 conditions were analyzed using an unpaired $t$-tests, where ${ }^{*}$ indicates $P<0.05$ compared with control. In the case of the cell size experiment, a 2tailed $t$-test with Welch's correction was used to compare the differences between conditions. Experiments with 4 or more conditions were analyzed using a 1-way ANOVA, with Tukey's test for multiple comparison, where * indicates $P<0.05$ compared with control, and ${ }^{* *}$ indicates $P<$ 0.05 compared with treatment. All statistical analysis was done using GraphPad Prism 6 software.

\begin{abstract}
Acknowledgements
This work was supported by the Natural Sciences and Engineering Research Council (NSERC) Canada, through a Discovery Grant to J.W.G. and a studentship to J.T.F. Seed funding was provided by the Children's Hospital Foundation of Manitoba. W.D.J. is funded by a RIG grant from Athabasca University and T.L.I. is funded through the SFRG and UCRP programs from the University of Manitoba. G.M.H. and J.W.G. are supported by the Heart and Stroke Foundation of Canada and are members of the DEVOTION Research Cluster. G.M.H. is a Canada Research Chair in Molecular Cardiolipin Metabolism. W.M., J.T.F., and M.D.M. are supported by studentships from the Children's Hospital Foundation of Manitoba and Research Manitoba, M.D.M. received support from the DEVOTION research cluster. We wish to thank Dr. Tetsuaki Miyake for the NFAT-YFP plasmid and Caitlin Blaney for assistance with the neonatal hypoxia model and misoprostol injections.
\end{abstract}

\section{Author details}

${ }^{1}$ Department of Human Anatomy and Cell Science, University of Manitoba, Winnipeg, Canada. ${ }^{2}$ Children's Hospital Research Institute of Manitoba, Winnipeg, Canada. ${ }^{3}$ Department of Pharmacology and Therapeutics, University of Manitoba, Winnipeg, Canada. ${ }^{4}$ The Diabetes Research Envisioned and Accomplished in Manitoba (DREAM) Theme, Children's Hospital Research Institute of Manitoba, Winnipeg, Canada. ${ }^{5}$ Department of Psychology, University of Manitoba, Winnipeg, Canada. ${ }^{6}$ Department of Biological Science, University of Manitoba, Winnipeg, Canada. ${ }^{7}$ Faculty of Health Disciplines, Athabasca University, Edmonton, Canada. ${ }^{8}$ College of Nursing, University of Manitoba, Winnipeg, Canada

\section{Author contributions}

J.W.G., W.D.J., and T.L.I. conceived and coordinated the study. J.W.G., W.D.J., T.L. I., M.D.M., and J.T.F. wrote the paper. J.T.F. and M.D.M. designed and conducted most of the experiments. J.T.F. and M.D.M. were also responsible for data analysis and presentation. W.M. designed and conducted mitochondrial respiration experiments. D.C. performed myocyte isoloations and experiments, and provided assistance on gene expression experiments. Y.H. designed and conducted the RT-PCR experiments, and designed and constructed all novel plasmids. J.W.G. performed in silico work, including the 3D modeling and alignments. T.LI. and M.D.M. designed and conducted the in vivo hypoxia experiments. J.W.G., W.D.J., T.L.I, and G.M.H. were responsible for funding acquisition. All authors reviewed the results, edited, and approved the final version of the manuscript.

Conflict of interest

The authors declare that they have no conflict of interest. 


\section{Publisher's note}

Springer Nature remains neutral with regard to jurisdictional claims in published maps and institutional affiliations.

The online version of this article (https://doi.org/10.1038/s41420-018-0104-z) contains supplementary material, which is available to authorized users.

Received: 27 July 2018 Revised: 5 August 2018 Accepted: 5 August 2018 Published online: 21 September 2018

\section{References}

1. Douglas-Escobar, M. \& Weiss, M. D. Hypoxic-ischemic encephalopathy: a review for the clinician. JAMA Pediatr. 169, 397-403 (2015).

2. Neu, J. \& Walker, W. A. Necrotizing enterocolitis. N. Engl. J. Med. 364, 255-264 (2011).

3. Wang, H., Zhang, S. X. \& Hartnett, M. E. Signaling pathways triggered by oxidative stress that mediate features of severe retinopathy of prematurity. JAMA Ophthalmol. 131, 80-85 (2013).

4. Dakshinamurti, S. Pathophysiologic mechanisms of persistent pulmonary hypertension of the newborn. Pediatr. Pulmonol. 39, 492-503 (2005).

5. Armstrong, K., Franklin, O., Sweetman, D. \& Molloy, E. J. Cardiovascular dysfunction in infants with neonatal encephalopathy. Arch. Dis. Child. 97, 372-375 (2012).

6. Shastri, A. T., Samarasekara, S., Muniraman, H. \& Clarke, P. Cardiac troponin I concentrations in neonates with hypoxic-ischaemic encephalopathy. Acta Paediatr. 101, 26-29 (2012).

7. Greer, S. N., Metcalf, J. L., Wang, Y. \& Ohh, M. The updated biology of hypoxiainducible factor. EMBO J. 31, 2448-2460 (2012).

8. Carmeliet, P. et al. Role of HIF-1alpha in hypoxia-mediated apoptosis, cell proliferation and tumour angiogenesis. Nature 394, 485-490 (1998).

9. Kothari, S. et al. BNIP3 plays a role in hypoxic cell death in human epithelial cells that is inhibited by growth factors EGF and IGF. Oncogene $\mathbf{2 2}$, 4734-4744 (2003).

10. Azad, M. B. et al. Hypoxia induces autophagic cell death in apoptosiscompetent cells through a mechanism involving BNIP3. Autophagy 4, 195-204 (2008).

11. Mazure, N. M. \& Pouysségur, J. Atypical BH3-domains of BNIP3 and BNIP3L lead to autophagy in hypoxia. Autophagy 5, 868-869 (2009).

12. Zhang, L., Li, L., Liu, H., Borowitz, J. L. \& Isom, G. E. BNIP3 mediates cell death by different pathways following localization to endoplasmic reticulum and mitochondrion. FASEB J. 23, 3405-3414 (2009).

13. Zhang, J. \& Ney, P. A. Role of BNIP3 and NIX in cell death, autophagy, and mitophagy. Cell Death Differ. 16, 939-946 (2009).

14. Vande Velde, C. et al. BNIP3 and genetic control of necrosis-like cell death through the mitochondrial permeability transition pore. Mol. Cell. Biol. 20, 5454-5468 (2000).

15. Gordon, J. W., Shaw, J. A. \& Kirshenbaum, L. A. Multiple facets of NF-kB in the heart: to be or not to NF-KB. Circ. Res. 108, 1122-1132 (2011).

16. Hayden, M. S. \& Ghosh, S. Shared principles in NF-kappaB signaling. Cell 132, 344-362 (2008).

17. Hayden, M. S. \& Ghosh, S. Signaling to NF-kappaB. Genes Dev. 18, 2195-2224 (2004).

18. Zhong, H., Voll, R. E. \& Ghosh, S. Phosphorylation of NF-kappa B p65 by PKA stimulates transcriptional activity by promoting a novel bivalent interaction with the coactivator CBP/p300. Mol. Cell 1, 661-671 (1998).

19. Zhong, H., May, M. J., Jimi, E. \& Ghosh, S. The phosphorylation status of nuclear NF-kappa B determines its association with CBP/p300 or HDAC-1. Mol. Cell $\mathbf{9}$, 625-636 (2002).

20. Zhong, $H_{\text {., }}$ SuYang, $H_{\text {., }}$ Erdjument-Bromage, H., Tempst, $P$. \& Ghosh, S. The transcriptional activity of NF-kappaB is regulated by the IkappaB-associated PKAC subunit through a cyclic AMP-independent mechanism. Cell 89, 413-424 (1997).

21. Gang, H. et al. A novel hypoxia-inducible spliced variant of mitochondrial death gene Bnip3 promotes survival of ventricular myocytes. Circ. Res. 108, 1084-1092 (2011).

22. Diehl-Jones, W. et al. Human milk fortification increases Bnip3 expression associated with intestinal cell death in vitro. J. Pediatr. Gastroenterol. Nutr. 61, 583-590 (2015).
23. Ward, N. L. et al. Cerebral angiogenic factors, angiogenesis, and physiological response to chronic hypoxia differ among four commonly used mouse strains. J. Appl. Physiol. 102, 1927-1935 (2007).

24. Wallace, M. G., Hartle, K. D., Snow, W. M., Ward, N. L. \& Ivanco, T. L. Effect of hypoxia on the morphology of mouse striatal neurons. Neuroscience 147, 90-96 (2007).

25. Chae, I. G. et al. Generation of ROS by CAY10598 leads to inactivation of STAT3 signaling and induction of apoptosis in human colon cancer HCT116 cells. Free Radic. Res. 48, 1311-1321 (2014).

26. Ding, $Y$. et al. Ratiometric biosensors based on dimerization-dependent fluorescent protein exchange. Nat. Methods 12, 195-198 (2015).

27. Kelley, L. A., Mezulis, S., Yates, C. M., Wass, M. N. \& Sternberg, M. J. E. The Phyre2 web portal for protein modeling, prediction and analysis. Nat. Protoc. 10, 845-858 (2015).

28. Gordon, J. W. Regulation of cardiac myocyte cell death and differentiation by myocardin. Mol. Cell. Biochem. 437, 119-131 (2018).

29. Kwong, J. Q. \& Molkentin, J. D. Physiological and pathological roles of the mitochondrial permeability transition pore in the heart. Cell Metab. 21, 206-214 (2015).

30. Izzo, V., Bravo-San Pedro, J. M., Sica, V., Kroemer, G. \& Galluzzi, L. Mitochondrial permeability transition: new findings and persisting uncertainties. Trends Cell Biol. 26, 655-667 (2016).

31. Mughal, W. et al. A conserved MADS-box phosphorylation motif regulates differentiation and mitochondrial function in skeletal, cardiac, and smooth muscle cells. Cell Death Dis. 6, e1944 (2015).

32. Kwong, J. Q. et al. The mitochondrial calcium uniporter selectively matches metabolic output to acute contractile stress in the heart. Cell Rep. 12, 15-22 (2015).

33. Luongo, T. S. et al. The mitochondrial calcium uniporter matches energetic supply with cardiac workload during stress and modulates permeability transition. Cell Rep. 12, 23-34 (2015).

34. Szabadkai, G. et al. Chaperone-mediated coupling of endoplasmic reticulum and mitochondrial Ca2+ channels. J. Cell Biol. 175, 901-911 (2006).

35. De Stefani, D. et al. VDAC1 selectively transfers apoptotic $\mathrm{Ca} 2+$ signals to mitochondria. Cell Death Differ. 19, 267-273 (2011).

36. Zhao, Y. et al. An expanded palette of genetically encoded Ca2+ indicators. Science 333, 1888-1891 (2011).

37. Mughal, W. et al. Myocardin regulates mitochondrial calcium homeostasis and prevents permeability transition. Cell Death Differ. https://doi.org/10.1038/ s41418-018-0073-z (2018).

38. Chen, G. et al. The E1B $19 \mathrm{~K} / \mathrm{BCl}-2-$-binding protein Nip3 is a dimeric mitochondrial protein that activates apoptosis. J. Exp. Med. 186, 1975-1983 (1997).

39. Shimizu, S., Konishi, A., Kodama, T. \& Tsujimoto, Y. BH4 domain of antiapoptotic $\mathrm{BCl}-2$ family members closes voltage-dependent anion channel and inhibits apoptotic mitochondrial changes and cell death. Proc. Natl. Acad. Sci. U.S.A. 97, 3100-3105 (2000)

40. Wilkins, B. J. et al. Targeted disruption of NFATc3, but not NFATc4, reveals an intrinsic defect in calcineurin-mediated cardiac hypertrophic growth. Mol. Cell. Biol. 22, 7603-7613 (2002).

41. Wilkins, B. J. et al. Calcineurin/NFAT coupling participates in pathological, but not physiological, cardiac hypertrophy. Circ. Res. 94, 110-118 (2004).

42. Liu, Q., Chen, Y., Auger-Messier, M. \& Molkentin, J. D. Interaction between NFkB and NFAT coordinates cardiac hypertrophy and pathological remodeling. Circ. Res. 110, 1077-1086 (2012).

43. $\mathrm{Wu}, \mathrm{X}$. et al. Local InsP3-dependent perinuclear $\mathrm{Ca} 2+$ signaling in cardiac myocyte excitation-transcription coupling. J. Clin. Invest. 116, 675-682 (2006).

44. Chen, Y. et al. A nucleus-targeted alternately spliced Nix/Bnip3L protein isoform modifies nuclear factor KB (NFkB)-mediated cardiac transcription. J. Biol. Chem. 288, 15455-15465 (2013).

45. Yussman, M. G. et al. Mitochondrial death protein Nix is induced in cardiac hypertrophy and triggers apoptotic cardiomyopathy. Nat. Med. 8, 725-730 (2002).

46. Chen, Lf., Fischle, W., Verdin, E. \& Greene, W. C. Duration of nuclear NFkappaB action regulated by reversible acetylation. Science 293, 1653-1657 (2001).

47. Chen, L.-F. et al. NF-kappaB RelA phosphorylation regulates RelA acetylation. Mol. Cell. Biol. 25, 7966-7975 (2005). 
48. Kondo, K., Klco, J., Nakamura, E., Lechpammer, M. \& Kaelin, W. G. Inhibition of $\mathrm{HIF}$ is necessary for tumor suppression by the von Hippel-Lindau protein. Cancer Cell 1, 237-246 (2002).

49. Wu, J. et al. Improved orange and red $\mathrm{Ca} 2+$ indicators and photophysical considerations for optogenetic applications. ACS Chem. Neurosci. 4, 963-972 (2013).

50. Wu, J. et al. Red fluorescent genetically encoded Ca2+ indicators for use in mitochondria and endoplasmic reticulum. Biochem. J. 464, 13-22 (2014).

51. Wang, N. S., Unkila, M. T., Reineks, E. Z. \& Distelhorst, C. W. Transient expression of wild-type or mitochondrially targeted $\mathrm{BCl}-2$ induces apoptosis, whereas transient expression of endoplasmic reticulum-targeted $\mathrm{BCl}-2$ is protective against Bax-induced cell death. J. Biol. Chem. 276, 44117-44128 (2001).

52. Perciavalle, R. M. et al. Anti-apoptotic MCL-1 localizes to the mitochondrial matrix and couples mitochondrial fusion to respiration. Nat. Cell Biol. 14, 575-583 (2012)

53. Guan, B., Wang, T.-L. \& Shih, I.-M. ARID1A, a factor that promotes formation of SWI/SNF-mediated chromatin remodeling, is a tumor suppressor in gynecologic cancers. Cancer Res. 71, 6718-6727 (2011).
54. Perry, R. L. S. et al. Direct interaction between myocyte enhancer factor 2 (MEF2) and protein phosphatase 1alpha represses MEF2-dependent gene expression. Mol. Cell. Biol. 29, 3355-3366 (2009).

55. Du, M. et al. Protein kinase A represses skeletal myogenesis by targeting myocyte enhancer factor 2D. Mol. Cell. Biol. 28, 2952-2970 (2008).

56. Lee, M., Lapham, A., Brimmell, M., Wilkinson, H. \& Packham, G. Inhibition of proteasomal degradation of $\mathrm{Mcl}-1$ by cobalt chloride suppresses cobalt chloride-induced apoptosis in HCT116 colorectal cancer cells. Apoptosis 13 972-982 (2008)

57. Alizadeh, J. et al. Mevalonate cascade inhibition by simvastatin induces the intrinsic apoptosis pathway via depletion of isoprenoids in tumor cells. Sci. Rep. 7, 44841 (2017).

58. Pagiatakis, C., Gordon, J. W., Ehyai, S. \& Mcdermott, J. C. A novel RhoA/ROCKCPI-17-MEF2C signaling pathway regulates vascular smooth muscle cell gene expression. J. Biol. Chem. 287, 8361-8370 (2012)

59. Gordon, J. W. et al. Protein kinase A-regulated assembly of a MEF2.HDAC4 repressor complex controls c-Jun expression in vascular smooth muscle cells. J. Biol. Chem. 284, 19027-19042 (2009). 